\title{
Time-Accurate Unsteady Pressure Loads Simulated for the Space Launch System at Wind Tunnel Conditions
}

\author{
Stephen J. Alter; Gregory J. Brauckmann† Bil Kleb; \\ Christopher E. Glass;, Craig L. Streett $\$$ David M. Schuster ${ }^{\llbracket}$ \\ NASA Langley Research Center Hampton, Virginia 23681-2199
}

\begin{abstract}
A transonic flowfield about a Space Launch System (SLS) configuration was simulated with the Fully Unstructured Three-Dimensional (FUN3D) computational fluid dynamics (CFD) code at wind tunnel conditions. Unsteady, time-accurate computations were performed using second-order Delayed Detached Eddy Simulation (DDES) for up to 1.5 physical seconds. The surface pressure time history was collected at 619 locations, 169 of which matched locations on a 2.5 percent wind tunnel model that was tested in the $11 \mathrm{ft}$. $\times 11 \mathrm{ft}$. test section of the NASA Ames Research Center's Unitary Plan Wind Tunnel. Comparisons between computation and experiment showed that the peak surface pressure RMS level occurs behind the forward attach hardware, and good agreement for frequency and power was obtained in this region. Computational domain, grid resolution, and time step sensitivity studies were performed. These included an investigation of pseudo-time sub-iteration convergence. Using these sensitivity studies and experimental data comparisons, a set of best practices to date have been established for FUN3D simulations for SLS launch vehicle analysis. To the author's knowledge, this is the first time DDES has been used in a systematic approach and establish simulation time needed, to analyze unsteady pressure loads on a space launch vehicle such as the NASA SLS.
\end{abstract}

\section{Nomenclature}

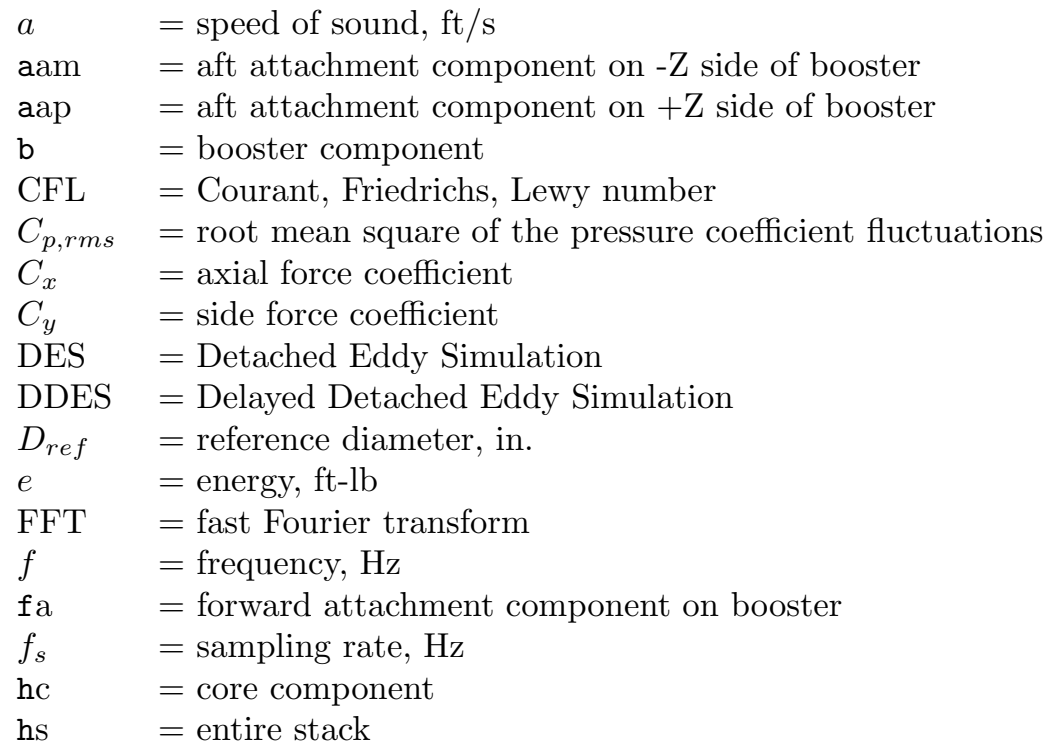

\footnotetext{
*Aerospace Research Engineer, Aerothermodynamics Branch; Senior Member AIAA

$\dagger$ Aerospace Research Engineer, Aerothermodynamics Branch; Associate Fellow AIAA

$¥$ Assistant Branch Head, Computational Aerosciences Branch; Senior Member AIAA

§Senior Aerospace Engineer, Computational Aerosciences Branch

\Senior Research Engineer, NASA Engineering Safety Center, Associate Fellow AIAA
} 


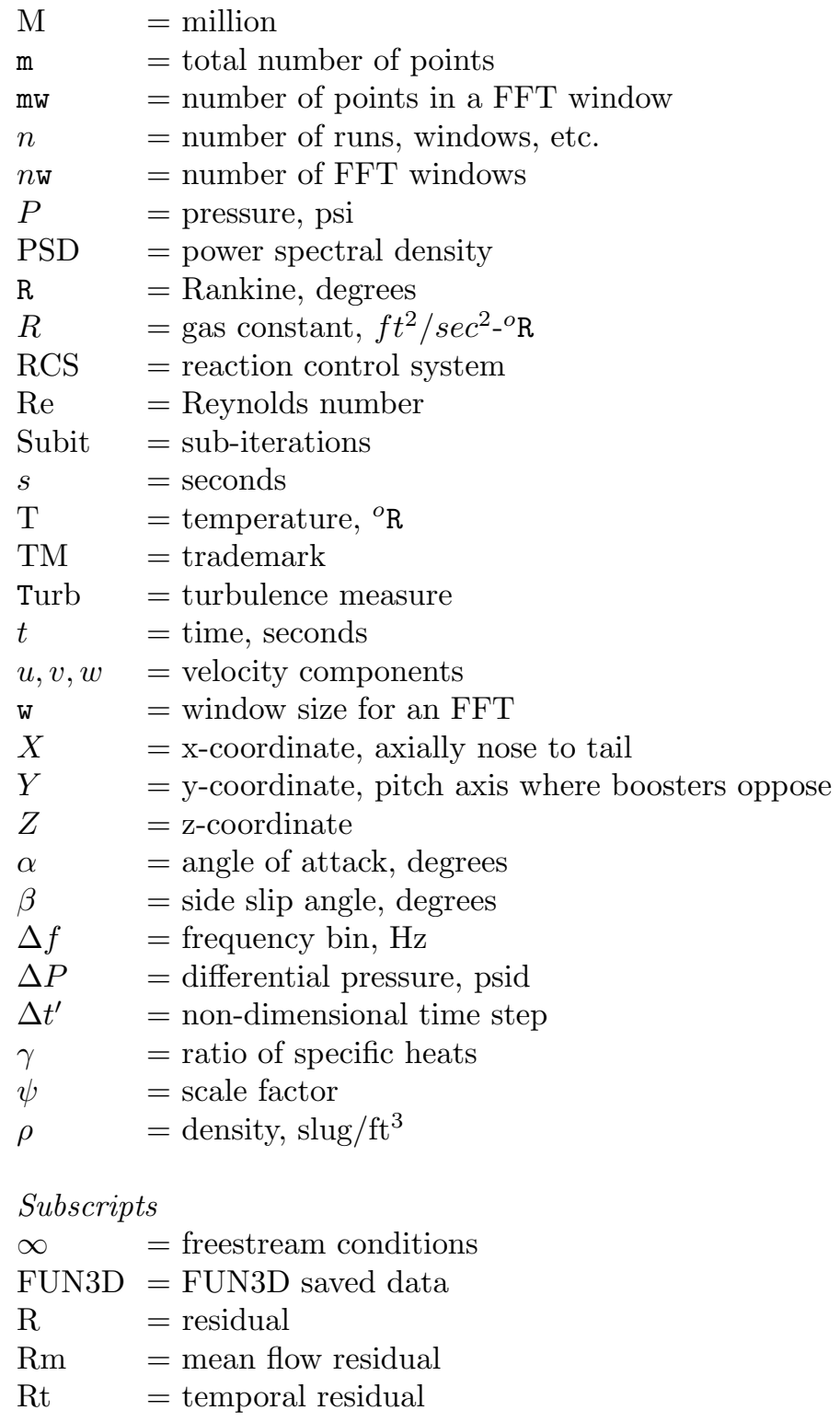

\section{Introduction}

NASA is developing the next generation of heavy lift launch vehicles known as the Space Launch System (SLS), designed to enable human exploration beyond low earth orbit. To reduce the development costs, previously developed hardware from the Space Shuttle and Constellation programs is being used to build initial configurations. The Block 1 SLS configuration uses Space Shuttle main engines to power a liquid propellant center core stage, augmented by two 5 -segment solid rocket boosters. SLS Block 1 is designed to lift 105 metric tons of payload and it is planned to evolve, as shown in Figure 1, to an ultimate capacity of least 130 metric tons. $^{1}$

Defining transonic buffet and aeroacoustic environments are significant design challenges for any launch vehicle. Here, buffet is defined as time-varying pressure fluctuations at frequencies below $100 \mathrm{~Hz}$ that can excite structural modes of the entire vehicle, while aeroacoustic fluctuations are at higher frequencies and are more localized in effect. Because buffet and aeroacoustic environments are closely tied to geometric details, they are highly configuration-specific. Until recently, experimental test methods ${ }^{3,4}$ have been the sole source for investigating such environments. The present study seeks to explore what state-of-the-art computational fluid dynamics (CFD) can offer in predicting buffet fluctuating pressure environments. 


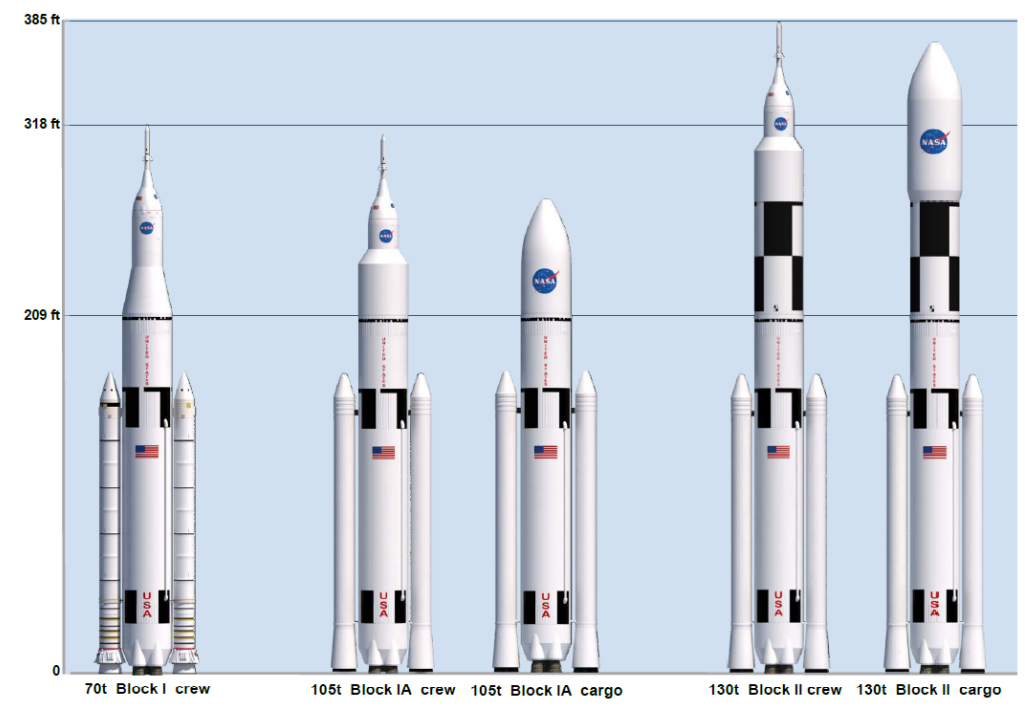

Figure 1. SLS variants (figure from a previous report ${ }^{2}$ ).

CFD is currently not a viable alternative to wind tunnel testing in developing buffet forcing functions for structural analyses. ${ }^{5}$ Accurate predictions with the temporal and the geometric resolution necessary to capture low frequency fluctuations, less than $100 \mathrm{~Hz}$ are difficult to produce with today's CFD. Typical Reynolds Averaged Navier-Stokes (RANS) simulations eliminate the frequency spectrum of the buffet condition in the low frequency range. Due to the length scale required to resolve buffet conditions, detached eddy simulations ${ }^{6}$ (DES) are better suited to resolve the unsteady pressure environment. Accurate DES computations can be performed with either high-order, low-dissipation schemes on typical RANS mesh densities, ${ }^{7,8}$ or lower-order schemes with significantly higher mesh resolution than standard RANS computations. ${ }^{9}$ Additionally, when performing DES computations, the time step required to maintain accuracy tends to be smaller than RANS. Thus, to obtain sufficient data to predict launch vehicle buffet environments using CFD, long run times on dense meshes are needed to resolve the desired frequency spectrum.

This paper discusses the use of CFD to predict the unsteady flow environments for launch vehicles. It focuses on the transonic flight regime where the largest buffet environments are typically encountered. An unstructured mesh flow solver is used to compute buffet environments on an SLS Block 1 cargo configuration. The data generated in this study were assessed in both time and frequency domains at selected locations on the vehicle. Wind tunnel test data for this configuration exists and have been used for comparison with computed data to assess the accuracy of the flow solutions. Mesh resolution and time step studies were performed to determine the best practices for the intended goal.

The main goal of this present study was to explore the current CFD capability to predict these environments, and define best practices for obtaining accurate and converged solutions. The CFD technique herein was applied in another study that sought to reduce elevated root mean squared (RMS) pressure levels in certain regions of the SLS by changing the booster nose shapes. ${ }^{10}$ The practices identified in this report can be utilized to improve the vetting of design changes, reduce adverse frequency-based loading of flight vehicles, and lead to the development of computationally-based buffet forcing functions and aeroacoustic environments.

\section{Problem Description}

This paper focuses on computing the fluctuating pressure environment of the cargo variant of the Block 1 SLS, as shown in Figure 1. A delayed, detached eddy simulation (DDES) CFD solution of the vehicle was conducted using an unstructured mesh code described in subsequent sections. For DDES, the DES limiter is used to delay the use of DES, enabling use of RANS in areas of thicker boundary layers. This effort focused on Mach numbers in the transonic flight range between Mach 0.9-1.2, and angles of attack ranging from 0 to 4 degrees, at unit Reynolds numbers from six million to 90 million per foot. A surface model of the vehicle analyzed along with a definition of the angle of attack are shown in Figure 2. 


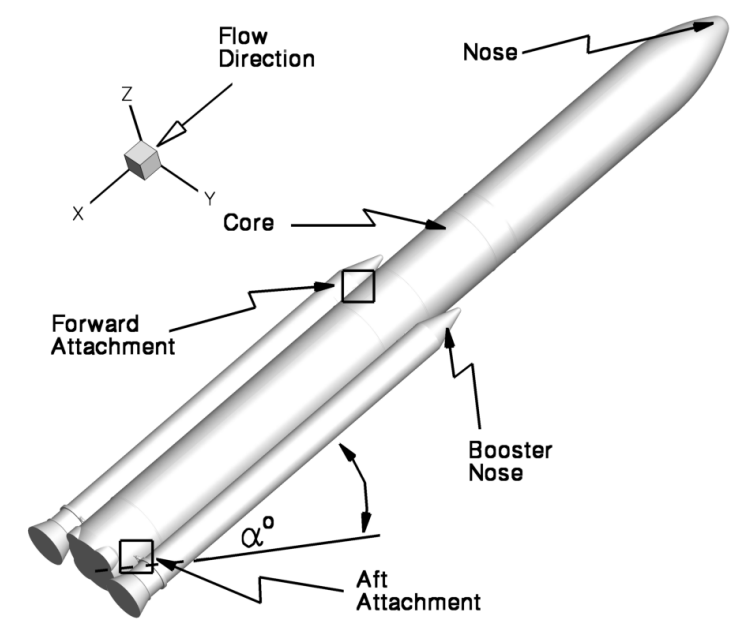

Figure 2. Baseline SLS stack with coordinate system used for computations.

The vehicle coordinate system origin is on the core axis and well ahead of the nose; but for clarity, the coordinate axes are offset in Figure 2 and are shown for orientation purposes only. The X-coordinate extends down the body from the nose to the base. The Z-coordinate is tangential to the pitch plane $(\alpha)$, so that the boosters are in the X-Y plane. The Y-coordinate extends from the core symmetry plane located between both boosters toward the right hand booster when looking forward on the vehicle. The angle of attack $(\alpha)$ is set when the body is rotated in the $\mathrm{X}-\mathrm{Z}$ plane about the $\mathrm{Y}$-axis, and side slip $(\beta)$ is located in the $\mathrm{X}-\mathrm{Y}$ plane when the vehicle is rotated about the Z-axis.

Wind tunnel data were obtained in NASA Langley's Transonic Dynamic Tunnel ${ }^{11}$ (TDT), and Ames' Unitary Plan Wind Tunnel (UPWT) complex ${ }^{12,13}$ for a significant breadth of these conditions. To date, reports for this data have not been published. Best practices from a supersonic retro-propulsion (SRP) study $^{14}$ were applied to the computation of unsteady loads on the SLS obtained from a TDT wind tunnel test. This was done as a feasibility study, which provided adequate accuracy of unsteady load comparisons. Based on the success of those comparisons, predictions of UPWT data were made for a future wind tunnel test. The methodology presented in this paper originated with the SRP best practices, but only computational and UPWT experimentally obtained data are compared in this paper. The analyzed configuration shown in Figure 2 has been modified from the actual flight vehicle to facilitate wind tunnel and CFD modeling. The geometry was reduced in complexity from the wind tunnel model through the removal of fasteners, booster separation motors, horizontal rib stiffeners, and engine nozzles to generate the CFD geometry. This shape is referred to as the ascent acoustics model for the Ames Acoustics Test, and is described in greater detail in the remainder of this paper. Sections on the flow solver and mesh generation methods, computational resources and techniques, post-processing data and baseline configuration results are presented.

\section{Flow Solver}

Computational analyses of the unsteady flowfield for the SLS stack were performed using the Fully Unstructured Three-Dimensional (FUN3D) software. ${ }^{15,16}$ Originally developed at the NASA Langley Research Center as a research code in the 1980s, it has become a workhorse for computations throughout flowfield regimes that range from incompressible to hypersonic flow. For this study, FUN3D was run using a modified Delayed Detached Eddy Simulation (DDES) ${ }^{17}$ hybrid RANS/Large Eddy Simulation model with a selectively-dissipative version of Edwards Low-Dissipation Flux Splitting Scheme inviscid flux function, ${ }^{18}$ a modified Van Albada limiter, ${ }^{19,20}$ and a Spalart-Allmaras near-wall model. ${ }^{21}$ Node-based conservative variables are computed by driving a second-order accurate spatial residual to steady-state with a point-implicit iterative method. The solution was advanced in time with an optimal second-order modified backward difference formula scheme. ${ }^{17}$ Ideally, the solution would be advanced to the next time step when the mean flow residuals dropped an order of magnitude below the temporal error estimate. In practice, the solution was advanced when the mean flow residuals and the temporal error estimates were similar. 
To facilitate efficient computing, both time step and mesh density sensitivities were evaluated based on convergence criteria. The basis for these computations will be discussed in the results section where computing the baseline data is addressed. All computations, including the sensitivity studies, were based on the same procedure. Metrics for assessing convergence of a time accurate simulation are complicated because of the difficulty in getting mean flow residuals an order of magnitude less than the temporal error estimate. The solution computed in this work does show a drop in the mean flow residuals to levels that are at least seven orders of magnitude smaller than the initial values. However, there is no asymptotic decay of the residuals because of the unsteady nature of the flowfield. So an alternative approach had to be used to determine convergence of the solution. Generally, the procedure used was to identify the frequency of peak power seen in the power spectral densities (PSDs), and to minimize any discrepancy between CFD and wind tunnel values. This is distinctly separate from the computational sub-iteration convergence. In the absence of wind tunnel data, grid convergence studies with time step sensitivity analyses would have been done. Having the experimental data reduced the work necessary to determine the best time step and mesh for the computation. The time step used for a computation was considered appropriate when there was consistency between the PSD of computationally and experimentally obtained data. Deviations from that time step were used to capture the sensitivity to accurately predict PSDs.

\section{Mesh Generation Method}

The surface and volume meshes for this study were generated with the NX computer aided design (CAD) tool, ${ }^{22}$ Grid Express (GridEx), ${ }^{23,24}$ and the advancing front local reconnection software (AFLR3) ${ }^{25,26}$. All solid manifold representations of geometry were generated by the NX CAD tool. The original data used as input for the grid generation process either originated from NX itself, by using the parametric modeling features, or by loading in CAD parts from other CAD systems used by the SLS project engineers. Figure 3 shows an example of the NX interface with the simplified rigid body model (RBM) geometry displayed.

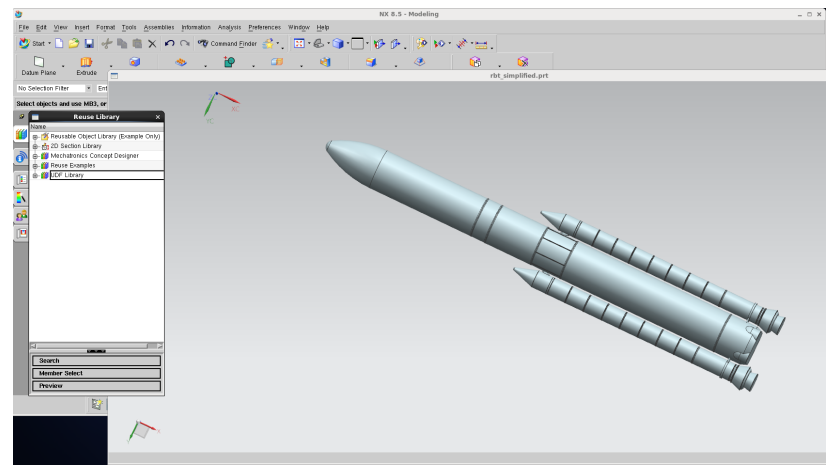

(a) NX.

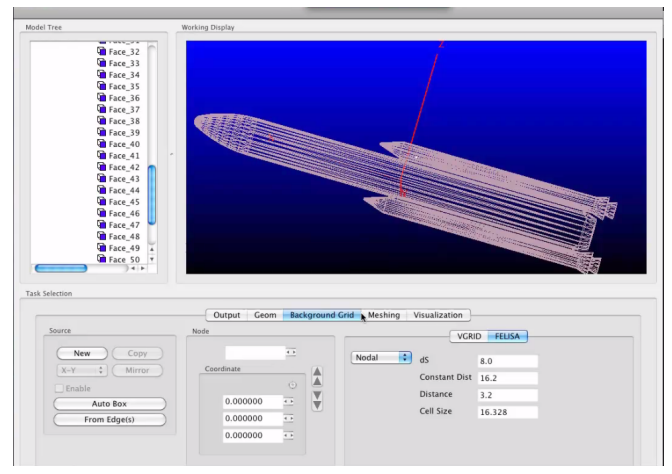

(b) GridEx.

Figure 3. RBM simplified model displayed in the NX CAD and GridEx software.

When completed in NX, a Parasolid part file was written for use with GridEx. GridEx is graphical user interface-driven software that enables the user to develop input data for the AFLR3 unstructured grid generator. AFLR3 uses FELISA ${ }^{27,28}$ or VGRID $^{29,30}$ mesh generators to develop surface grids. GridEx uses the same geometry kernel as NX for solid model construction, through an application programming interface (API) with the computational analysis programming interface (CAPrI) ${ }^{31}$ This API enables grids to be generated on the actual CAD description of models by using the native CAD geometry engine that created the data. The native CAD geometry eliminates data loss encountered if the initial graphics exchange specification (IGES) or the standard for the exchange of product model data (STEP) model descriptions were used. As shown in Figure 3, the GridEx software enables a user to define and set controlling parameters such as sources, for the mesh generation process.

Prior to meshing the surface of the vehicle, the wind tunnel SLS geometry is simplified to make the mesh generation easier and to reduce the size of the overall mesh that is produced. Such simplifications included removal of fuel feed lines and booster separation motors to make a smooth outer mold line as shown in Figure 4. In addition to the removal of the larger protuberances, simplifications were also made to the rings around the core, as shown in Figure 5. The locations of modified rings are identified within the red circles. 


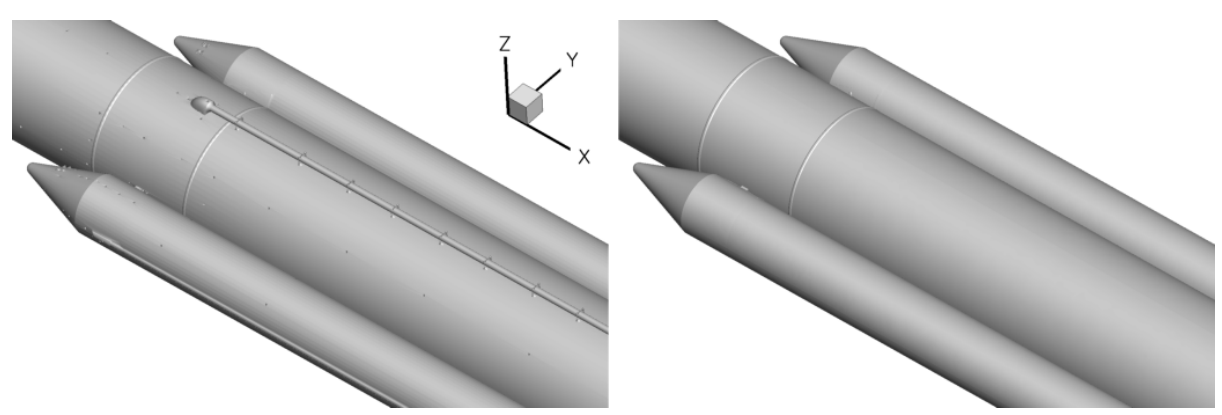

(a) Fuel line removal.

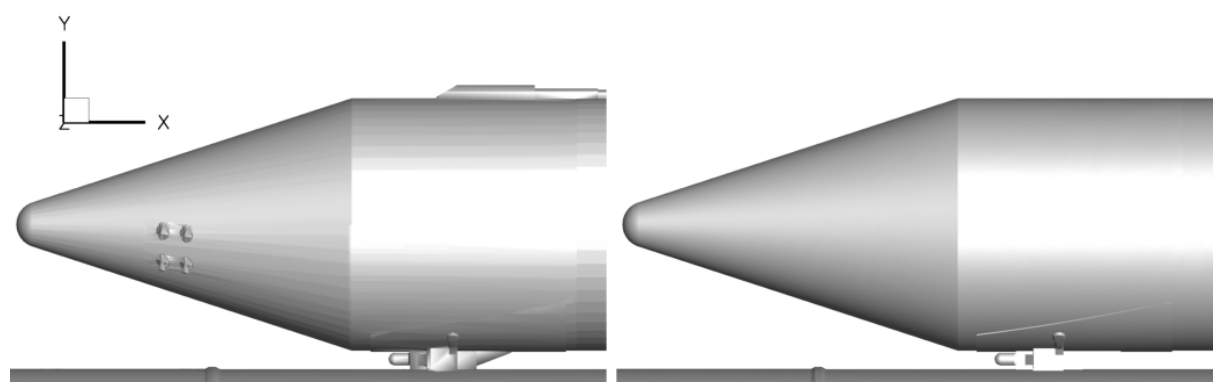

(b) Forward RCS removal.

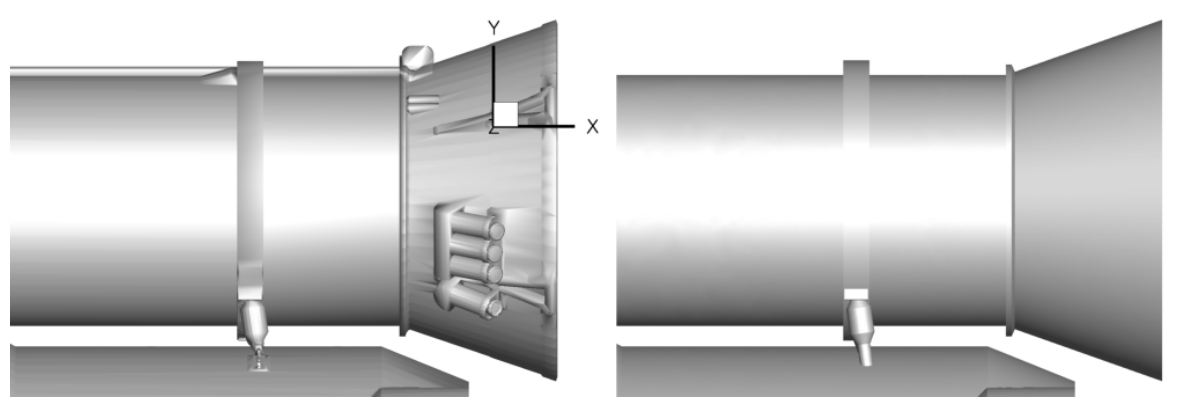

(c) Aft RCS removal.

Figure 4. Wind tunnel model simplifications for CFD applications; original geometry on left and modified on right.

For the viscous meshes in this study, approximately 50 prismatic cells extending orthogonally from the wall were used. The grid density was significant for the remainder of the domain, because the cells slowly grow to reach a distance of 10 times the body length in $\pm X,+Y$, and $\pm Z$ physical directions. A reference cell size determined all other cell size specifications in the mesh model. This enabled re-sizing of the mesh based on a single parameter for subsequent mesh resolutions, including those developed for a mesh sensitivity study discussed subsequently. In addition to the reference cell size, all geometries are scaled to the core diameter of the SLS configuration. Sources are used to impose grid clustering at surface features such as protuberances, surface intersections of vehicle parts such as core-to-booster attachment hardware, and coupling rings around the core and booster. Scaling by the diameter enables use of the same sources from geometry to geometry, thereby reducing the time to generate the mesh.

The end result of running GridEx is a batch script to generate the AFLR3 input surface meshes and volume source controls and AFLR3 is used to generate an unstructured volume mesh. Figure 6 shows a surface mesh for a typical geometry. The volume mesh is not shown in this paper due to the density required to accurately compute DDES solutions. The baseline mesh used for computations in this paper contained more than 35 million points and more than 200 million tetrahedral cells.

The process described above produces unstructured meshes for the SLS stack. To reduce complexity and in many cases the time to generate a mesh, only half of the vehicle is generated, using the pitch-plane for symmetry. When full body volume meshes are needed for computing, the FUN3D flow solver was used to 

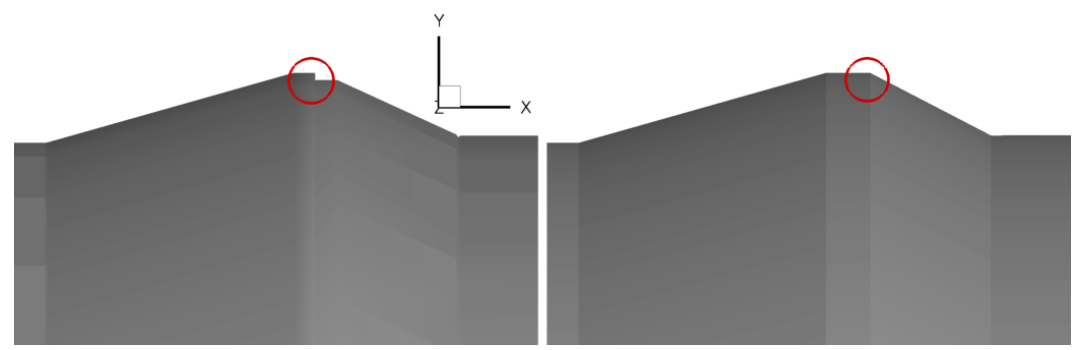

(a) Forward ring.

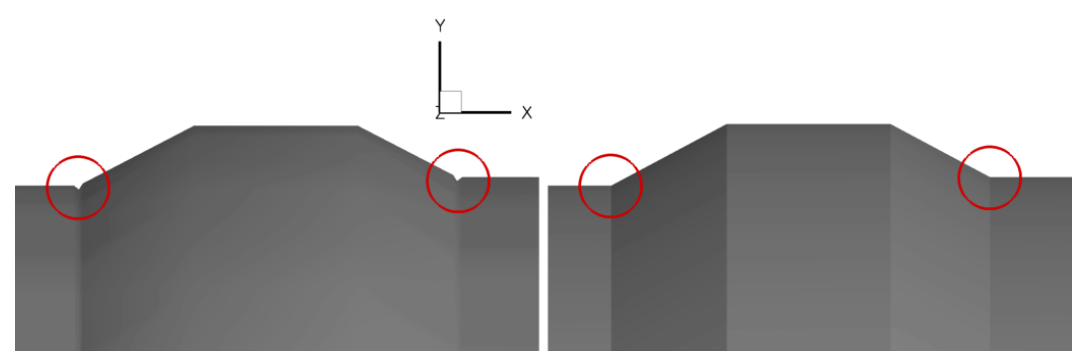

(b) Forward of booster forward attachment.

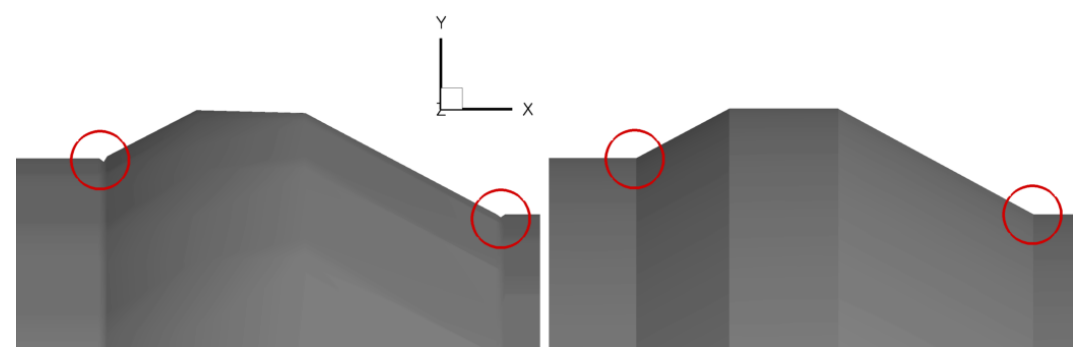

(c) Near aft booster attachment.

Figure 5. Wind tunnel model core, smaller ring simplifications; original geometry on left and modified on right.

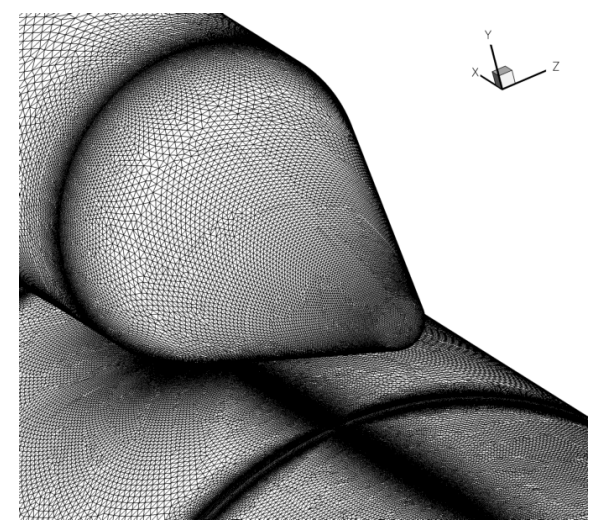

Figure 6. Baseline unstructured surface mesh from AFLR3.

mirror the vehicle mesh. This parallelized process is far faster than generating the full body mesh with the above sequential process. Mirroring the mesh with FUN3D is performed with command line options, e.g.,

mpiexec -np 50 nodet_mpi --mirror_y --write_mesh

where -np 50 specifies that 50 computing cores should be used, nodet_mpi is the FUN3D executable, --mirror_y instructs FUN3D to mirror the mesh about the X-Z plane, and --write_mesh will cause FUN3D to write out the mesh. A new boundary condition file will be produced as part of the process. 


\section{CFD Approach}

The solutions for the current study were begun as unsteady from the start of the computation. Based on past experience, ${ }^{14}$ proper flowfield initialization and a phased time step schedule gave the most robust and efficient start-up. First, the solution domain was initialized with freestream flow except for a pocket of quiescent gas around the vehicle, measuring a core diameter ahead and behind the core, and a radius of two body diameters from the $\mathrm{X}$-axis to form an encompassing cylinder. The initial stagnant fluid around the vehicle allows the inviscid flux functions within the CFD solver time to reduce the pressure without freestream velocities creating jump states that are difficult to solve by a linearized Riemann solver. Second, the non-dimensional time step, $\Delta t^{\prime}$, was ramped down to the final time step in stages to rapidly establish the mean flowfield. This was done in three phases, starting with 2,500 steps at a time step of $0.1,2,500$ steps at a time step of 0.01 , and finally a time step of 0.003 for all subsequent steps. For FUN3D, time is non-dimensionalized by the freestream speed of sound, $a_{\infty}$, and a length scale, which in this case is the core-stage base diameter, $D_{r e f}$, viz.,

$$
\Delta t^{\prime}=t \frac{a_{\infty}}{D_{\text {ref }}}
$$

So, for a non-dimensional time step of 0.1 , a freestream acoustic wave takes tens steps to travel one core-stage base diameter. The final time-step size was set to provide approximately 200 steps per cycle of the highest frequency of interest, as suggested in References 16 and 14. An upper limit of $60 \mathrm{~Hz}$ (2,400 Hz model scale) was assumed, resulting in a final non-dimensional time step of 0.003. Figure 7 shows a sample pressure trace for this phased start-up process, while Figure 8 shows a complete time history for 0.5 seconds, which required 12 days of computing on 2,500 Ivy Bridge cores $^{32}$ with the baseline mesh of $35 \mathrm{M}$ nodes.

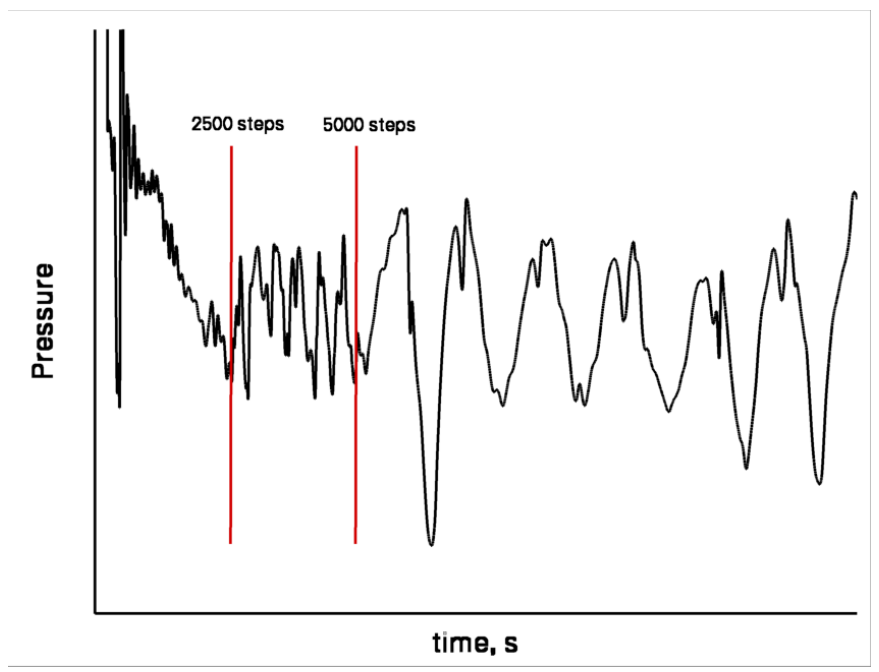

Figure 7. Unsteady surface pressure signature near the forward attachment for the initial computing of a solution.

Here, the phased start-up is only a tiny fraction in the beginning. The spikes in residual histories shown in Fig 8(a) are the result of the sub-iteration linear solver failing to converge one of the residuals of a particular time step and had no discernible effect on the computations. Figure 8(b) shows the body force and moment time history.

Other parameters used to control the accuracy of the solver include the number of pseudo-time subiterations between time steps. Ideally, the sub-iteration count should be sufficient to lower the mean flow residuals an order of magnitude less than the temporal error estimate. Unfortunately, due to the small time step size and the linear solver performance, it was often not possible to drive the mean flow residuals below the temporal error estimates. However, as shown in Figure 9, several different counts of sub-iteration at a constant Courant-Fredrichs-Lewy (CFL) number and different rates of advancing the solution with CFL at a constant sub-iteration count, were used to find the best compromise between mean flow residual error and temporal error estimates. For the computations in this paper, seven sub-iterations per time step were used at a CFL of 20. Each time the mesh density or flow conditions changed, subsequent sub-iteration count and CFL studies were performed. 


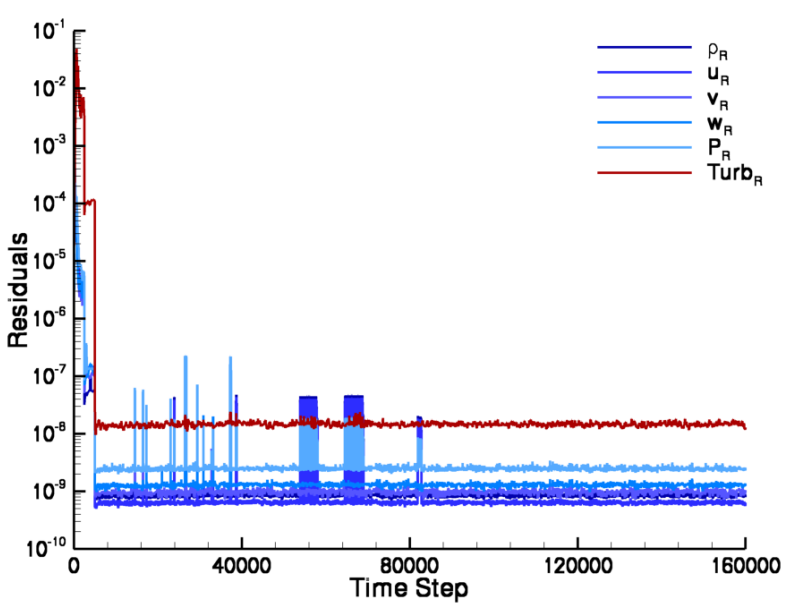

(a) Residual history.

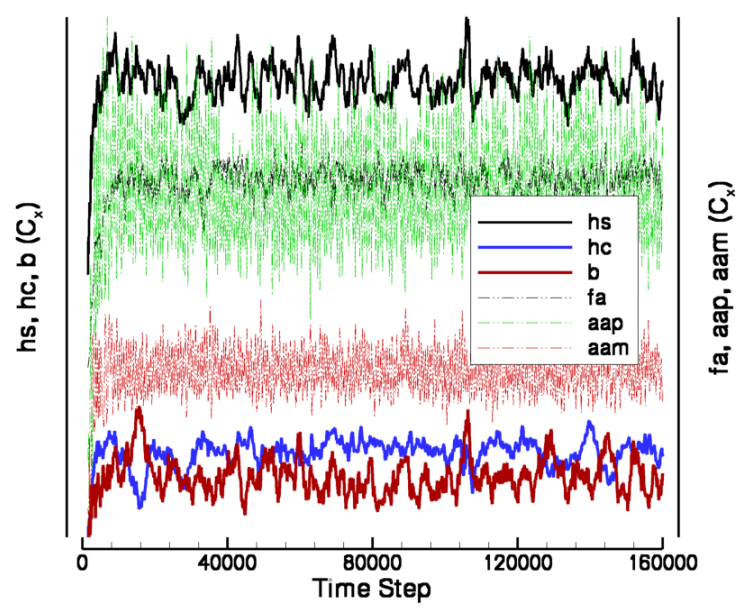

(b) Force and moments.

Figure 8. Solver performance and body force and moments for a 0.5 second simulation.

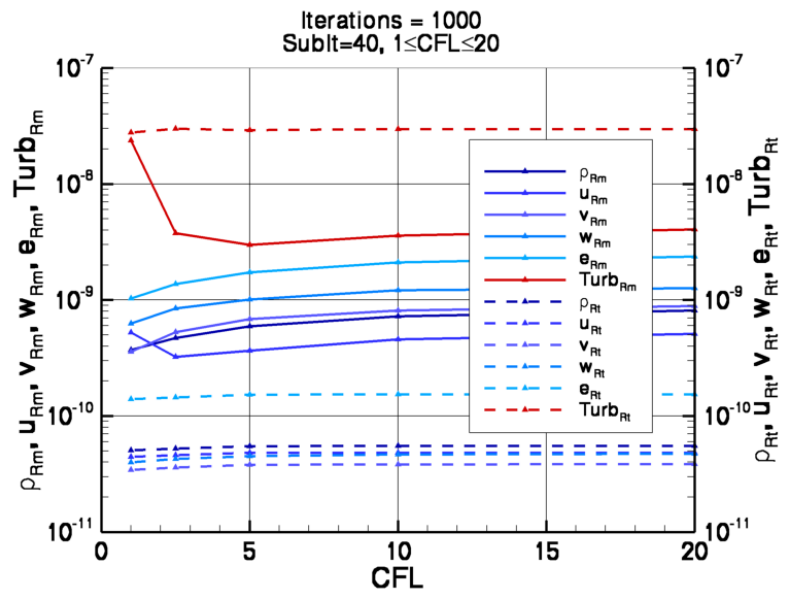

(a) CFL variation.

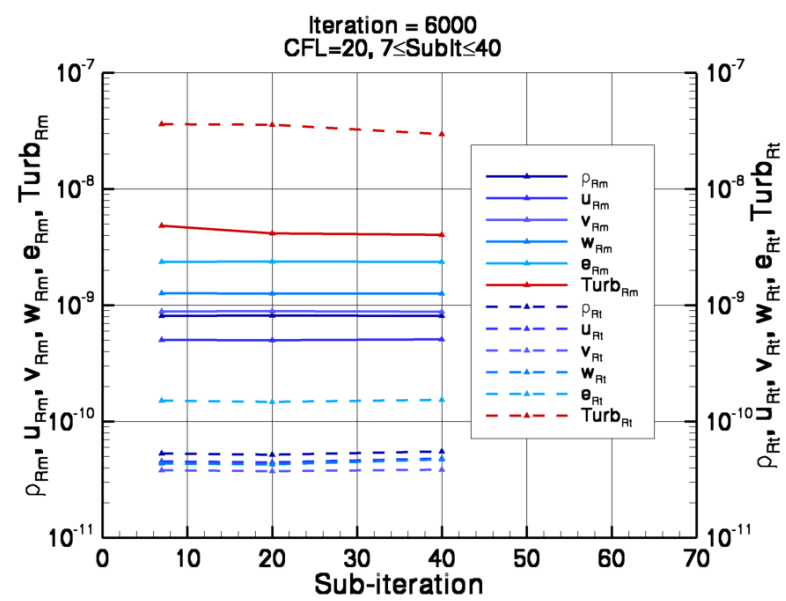

(b) Sub-iteration variation.

Figure 9. Mean flow and temporal error flowfield variables residual sensitivity to CFL and sub-iterations.

Initially, only a quarter vehicle was used to gain experience with this configuration and to provide rapid feedback with respect to grid point distributions. Due to the symmetry imposed, the wake behind the forward booster attachment was forced to be symmetric. When this symmetry restriction was removed by using the half-body domain, the flowfield behind the attach hardware was asymmetric. The asymmetric flowfield is believed to be the correct solution, as shown by the asymmetric flowfield patterns in Figure 10. Thus, the computations that were used for this study were predominantly half vehicle, with an X-Z plane of symmetry that gave one complete booster and associated core-booster attachment region. To check the effect of this single-symmetry assumption, full body configurations were also run for some cases. For the full three-dimensional cases, asymmetric effects were not present, both at angles of attack of zero and four degrees. Thus, half body cases that include the entire booster were used for the data in this report.

\section{Resources}

Performing accurate DDES computations requires meshes of significant density to resolve local eddies in the viscous regions of the flowfield. ${ }^{6}$ With a baseline mesh of more than 35 million points, solutions with sufficient time steps require the use of high-end supercomputing resources. All computations for this work 


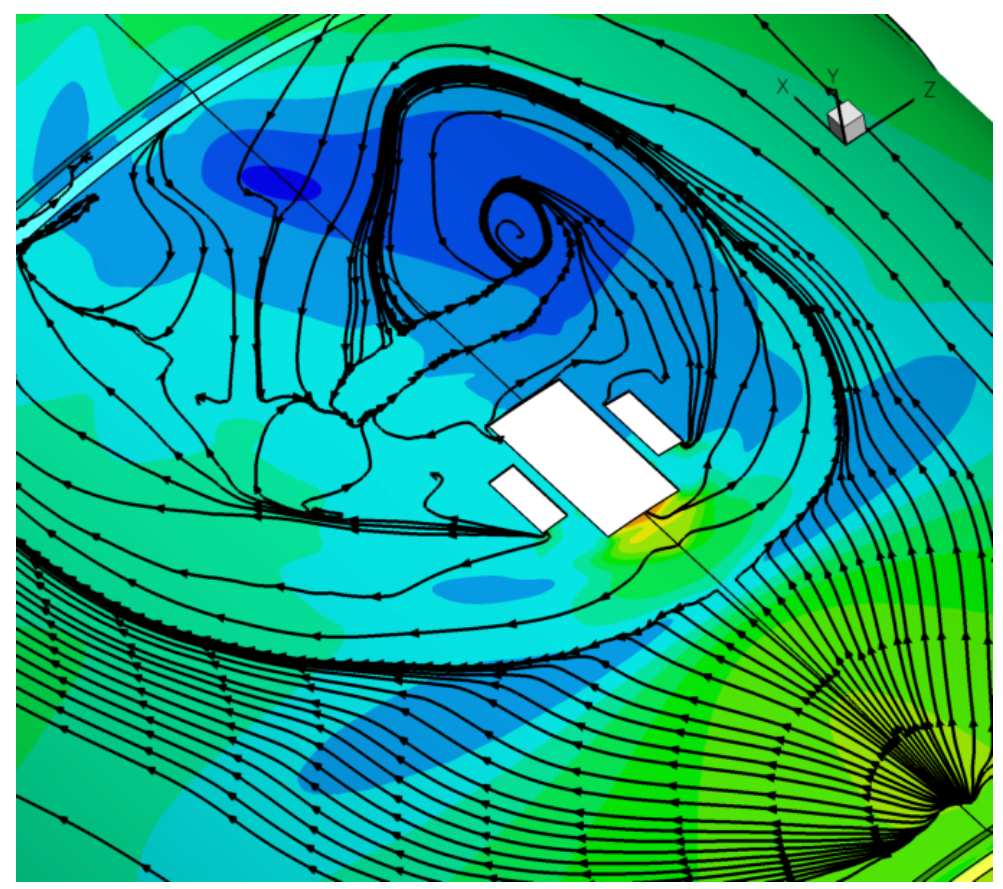

Figure 10. SLS core forward attachment region contoured with pressure illustrating asymmetric flowfield.

were performed on the NASA Advanced Supercomputing (NAS) Pleiades supercomputer, ${ }^{33}$ which has a heterogeneous combination of Westmere, ${ }^{\mathrm{TM}}$ Sandy Bridge, ${ }^{\mathrm{TM}}$ and Ivy Bridge ${ }^{\mathrm{TM}}$ Intel ${ }^{\circledR}$ processors that contain two sockets per node and eight, twelve, and twenty cores per socket, respectively. For this study, runs were made with between 14,000 and 28,000 mesh points per computing core depending on availability and priority of the core type. Different core types were used to minimize the time delay between a solution finishing and subsequently restarting. Note that for runs with a large number of cores, the mean time between failures goes up as the number of computing cores is squared; and so a larger number of points per computing core, up to 50,000 points per core, can improve stability at the expense of wall time. A failure is noted when a compute node fails to communicate with the master compute node.

The overall computing time, CPU_time, required to accurately predict unsteady pressures for the solutions in this paper, is determined by Eq. (2),

$$
\text { CPU_time }=\sum_{n=1}^{\text {run }_{\max }} \text { wall__clock }_{n} \times \# \text { _of_cores }{ }_{n} \times \psi_{n}
$$

where run $_{\max }$ is the total number of runs with each run defined by a set number of time steps, wall_clock $n$ is the number of hours per run, \#_of_cores $n$ is the number of computing cores, scaled by $\psi_{n}$, which is normalized by the NAS benchmark flow solvers computing efficiency of an Ivy Bridge ${ }^{\mathrm{TM}}$ processor. For the baseline case highlighted in this paper, the runs used to obtain 1.5 seconds of simulated time are listed in Table 1.

A total of 815,000 time steps was computed. Subtracting the initial 15,000 time steps from this count to remove effects of transient data in the computation, results in a total time of 1.5396 simulated seconds at $1.9245 \times 10^{-6}$ seconds per time step. Using Eq. (2), a total of 2817756.7 computing core hours was required to compute this case. Using the number of computational points per core limit of 12,500 , the optimum time computing this case using the fastest processors available at the time of the computations, the Ivy bridge $^{\mathrm{TM}}$ core, would have been 42 days. The actual time to compute this case was 67 days. The disparity arises from using different Intel ${ }^{\circledR}$ core types. 
Table 1. Runs required to simulate 1.5 seconds of a flowfield over the SLS.

\begin{tabular}{rrcrrrr} 
& Time & CPU & Number of & & Wall Clock & Simulated \\
Run & Steps & Type & Cores & $\psi$ & Hours & Time (sec.) \\
\hline 1 & 2,500 & Sandy Bridge & 1,280 & 0.903 & 1.7 & 0.1603750 \\
2 & 2,500 & Sandy Bridge & 1,280 & 0.903 & 1.4 & 0.0160375 \\
3 & 25,000 & Sandy Bridge & 1,280 & 0.903 & 53.1 & 0.0481125 \\
4 & 10,000 & Nehalem & 1,280 & 0.794 & 42.6 & 0.0192450 \\
5 & 20,000 & Nehalem & 1,280 & 0.794 & 89.1 & 0.0384900 \\
6 & 27,000 & Nehalem & 1,280 & 0.794 & 118.6 & 0.0519615 \\
7 & 13,000 & Nehalem & 1,280 & 0.794 & 56.2 & 0.0250185 \\
8 & 30,000 & Nehalem & 1,584 & 0.794 & 114.2 & 0.0577350 \\
9 & 30,000 & Nehalem & 1,584 & 0.794 & 114.3 & 0.0577350 \\
10 & 35,000 & Westmere & 1,992 & 0.661 & 62.8 & 0.0673575 \\
11 & 35,000 & Westmere & 1,992 & 0.661 & 64.1 & 0.0673575 \\
12 & 60,000 & Ivy Bridge & 2,500 & 1.000 & 70.9 & 0.1154700 \\
13 & 60,000 & Ivy Bridge & 2,500 & 1.000 & 83.2 & 0.1154700 \\
14 & 60,000 & Ivy Bridge & 2,500 & 1.000 & 75.5 & 0.1154700 \\
15 & 60,000 & Ivy Bridge & 2,500 & 1.000 & 74.2 & 0.1154700 \\
16 & 60,000 & Ivy Bridge & 2,500 & 1.000 & 74.8 & 0.1154700 \\
17 & 60,000 & Westmere & 1,992 & 0.661 & 108.6 & 0.1154700 \\
18 & 60,000 & Ivy Bridge & 2,500 & 1.000 & 89.7 & 0.1154700 \\
19 & 45,000 & Ivy Bridge & 2,500 & 1.000 & 88.6 & 0.0866025 \\
20 & 60,000 & Ivy Bridge & 2,500 & 1.000 & 100.3 & 0.1154700 \\
21 & 60,000 & Sandy Bridge & 2,000 & 0.903 & 111.2 & 0.1154700 \\
\hline Total & 815,000 & & & & 1595.1 & 1.7352575
\end{tabular}

\section{Post-Processing}

The intent of this work is to compliment wind tunnel experimentation with comprehensive global data for volume and surface properties that can be used in design and assessment studies. In the flow simulations performed with the CFD approach identified above, time-accurate unsteady pressure data is collected for a considerable length of simulated time. At the outset of this study, approximately 0.25 seconds of simulated time was collected. As the study progressed, it became apparent that longer simulation times were needed to compare with wind tunnel data, which was typically collected for 10 seconds. Although 10 seconds of simulated time was not possible due to the time required for the CFD process, as much as 1.1 seconds of simulated time was collected for a baseline computation. This data was then compared with wind tunnel data to check the accuracy of the CFD.

During the flowfield computations, the FUN3D code saves a variety of data for analysis. Five different sets of data were written, including aerodynamic parameters (e.g., pressure, velocity, density) for the wall boundaries, the X-Y plane, the location of the sonic condition in the flowfield, as well as monitor point pressure, and force and moments based on seven different components of the SLS stack. The wall boundary, $\mathrm{X}-\mathrm{Y}$ plane, and sonic condition data were written every 200 time steps, while the remaining data was written every time step.

The time-varying data analyzed in this paper is difficult to illustrate in static images. Thus, pressure, density, and temperature animations from the wall boundaries, X-Y plane, and sonic condition data were used to understand the flow physics. While animations cannot be shown in printed papers, typical plots at instantaneous times of shock structures and surface streamlines content were made to evaluate the results of the CFD. In addition to these plots, comparisons of additional derived data are made to evaluate the accuracy of the computations, including surface RMS of $C p$, frequency content, histograms, and coherence. 
Comparing simulated time and actual time is not easily done, because frequencies are difficult to distinguish in time-series plots between CFD and experimentally collected data. Instead, power spectral densities and other measures such as histograms of differential pressure and longitudinal coherence can be compared, but the unsteady pressure data computed has to be post-processed similarly, to make such a comparison. The pressure data that is written by FUN3D is non-dimensionalized by the freestream dynamic pressure with respect to the freestream speed of sound. Thus, equation 3 is used to convert the FUN3D pressure output to a differential pressure, in units of pounds per square inch (psid). Using this measure, PSDs of both CFD and experimental results can be calculated using Fast Fourier Transforms (FFT) methods.

$$
\Delta P=P_{\infty}\left(P_{\mathrm{FUN} 3 \mathrm{D}} * \gamma-1\right)
$$

Pressure fluctuations around a mean value are showin in Figure 11. PSDs are computed in order to look at the frequency content of this data. A single-window FFT can not guarantee the estimate of the power in a given frequency band is converged, so an average-based technique such as Welch's method is used. The data is divided into overlapping windows or blocks ( $75 \%$ overlap in our case), the PSDs for each window are computed and then averaged together.

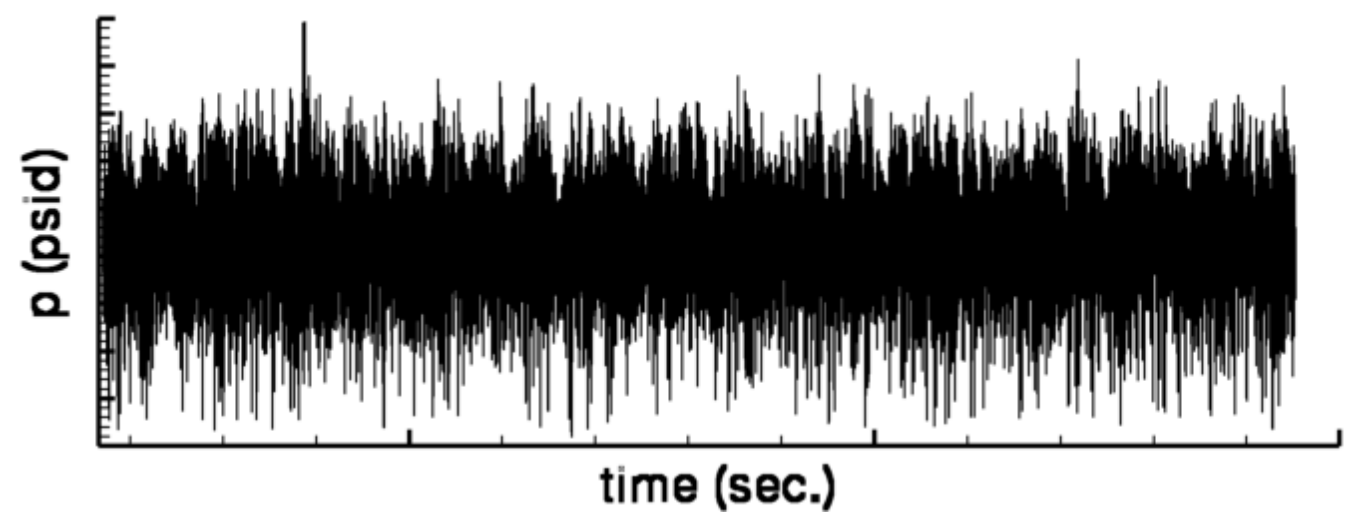

Figure 11. Typical unsteady pressure data collected over 1.5 seconds of simulated time.

$$
\Delta f=\frac{f_{s}(n \mathrm{w}+3)}{4 \mathrm{~m}}=\frac{f_{s}}{\mathrm{mw}}
$$

The frequency resolution, or FFT bin width, is determined from Eq. (4), derived from Welch's method. ${ }^{34}$ In Eq. 4, the total number of time steps available is $\mathrm{m}, f_{s}$ is the sampling rate, $\mathrm{mw}$ is the number of time steps in each window, $n \mathrm{w}$ is the number of windows, and $\Delta f$ is the frequency resolution, or bin width.

Increasing the number of windows (increasing the number of averages) drives down the variance of the estimated power in a frequency bin, but at the expense of a larger bin size, or reduced frequency resolution. Thus, more simulation time is required to generate the additional windows of data that will result in a decrease in the variance for a specified frequency resolution.

Histograms of $\Delta \mathrm{P}$ are generated by subdividing the range of differential pressure values into multiple discrete ranges or bins. Histograms count the number of occurrences of a value in a given range. Data from CFD and wind tunnel-collected measurements are used to produce two separate histograms. This is done so that the distribution in pressure difference over the collection time of the data can be compared between the CFD and the wind tunnel. For each histogram, the range of differential pressure is subdivided into 100 equally sized bins, but plotted such that the range is identical. Thus, some plots may appear compressed and some elongated, but this ensures that one-to-one comparisons between CFD and wind tunnel data can be done easily. If the distributions are close, it is assumed that the CFD is capturing the physics of the experiment. Histograms are especially useful to indicate bi-modal flows, containing both separated and attached flow characteristics. This comparison will be shown in the results section.

The final comparison is the longitudinal coherence. Coherence, or spectral coherence, is used to determine if any relationship exists between two signals. Magnitude-squared coherence is the ratio of the cross-correlation squared to the product of the two auto correlations, which occur between two locations. In 
the case of the computed or measured pressure data, the coherence is a measure of how well an upstream pressure measured signal will predict the signal at a point downstream. For two measurement points to be coherent, the signals do not have to be identical, but rather, if an upstream measurement varies and a consistent variation is measured at a downstream location, the signals exhibit coherence. If there is no general predictability between the two signals, then the coherence is zero; if there is a direct predictability, the coherence is unity. Generally, neither are the case, and the coherence measure of the predictability falls between 0 and 1 . The closer the measure is to unity, the more coherent the signal is to a downstream neighboring point.

By using the $C_{p, r m s}$, PSDs, histograms, and coherence, no single measure stands alone as a validation of accuracy. Collectively, they all are different measures that aid in the understanding of the accuracy of the CFD simulations. Thus, each is evaluated in the following results section of this report.

\section{Results}

The computations within this report focus on a baseline flow condition. This flow condition for the CFD was chosen to match nominal values from the SLS ascent acoustic wind tunnel test from the UPWT wind tunnel, and are as follows:

$$
\begin{array}{ll}
\text { Mach }=0.9 & \alpha=0^{\circ} \\
\text { Reynolds Number }=2.07 \times 10^{6} & \beta=0^{\circ} \\
\mathrm{T}_{\infty}=481.9^{\circ} \mathrm{R} & R=1716.49 \mathrm{ft}^{2} / \mathrm{sec}^{2}{ }^{\circ} \mathrm{R}
\end{array}
$$

Using FUN3D to compute this case required 590,000 time steps to accumulate sufficient simulation time, to perform all of the post-processing needed to compare to the wind tunnel data. Figure 12 shows the flow over the vehicle in the $\mathrm{X}-\mathrm{Z}$ plane for half the body which isolates the positive $\mathrm{Y}$ direction booster for an instant in time.

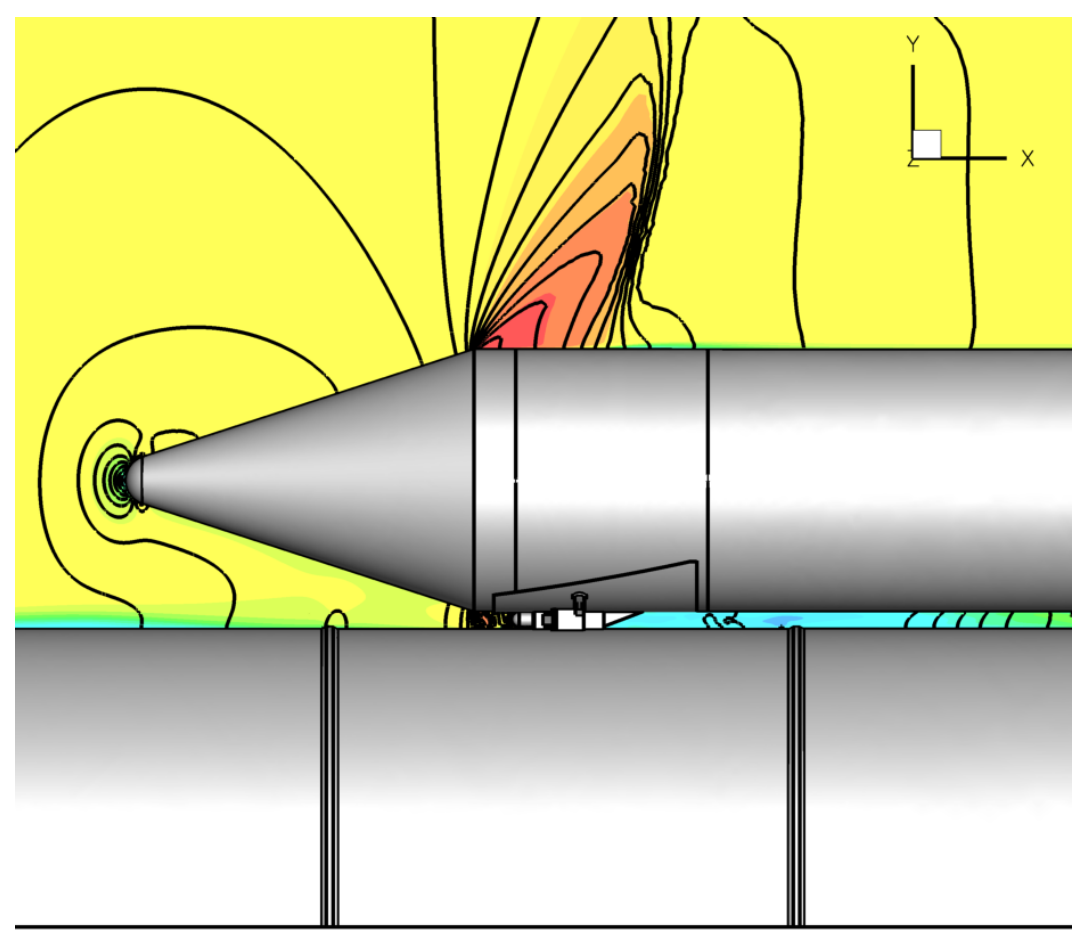

Figure 12. X-Y plane through booster and core with pressure contour and Mach lines.

In Figure 12, the lines are isobars of non-dimensional pressure, with streamwise velocity moving from left to right and color contoured on the plot. As would be expected, the Y-max corner of the booster near the attachment station to the booster shows a strong pressure gradient indicating an expansion wave. This region at the simulated Mach number, over expands resulting in formation of a shock wave downstream of 
the corner. The shock wave-boundary layer interaction then drives separation aft of the shock along the booster wall. A similar structure can be seen between the booster and the core, ahead of the attachment hardware. However, further upstream of the booster nose, there is also separation along the core. This separation is induced from the pressure wave building up at the front of the booster, but reattaches just before the booster nose at the core wall. This is a horseshoe vortex, shown in a side view in Figure 12, that is positioned upstream of the forward attachment hardware.

For a more global view of the flowfield, consider Figure 13, which illustrates (by the use of a Mach=1 iso-surface) the complex shock structures in the vicinity of the forward attach hardware. These three views represent consecutive time steps in an animation of the flowfield for a constant change in time steps between consecutive images. Just aft of the booster nose cone and cylinder, the flow reaches sonic speeds. A moving shock wave forms slowing the flow, but shock does not travel upstream to the nose cone junction. An expansion forms, causing the flow to again increase resulting in a second shock. This produces a region of compressed flow processed by the shock wave encompassing a region near the cone-cylinder junction to a distance approximately half a booster diameter down the booster from the junction. This type of flowfield has been studied in past years, ${ }^{35}$ and tends to be marginally stable. Any perturbation to this flow can cause an unsteady condition to occur. With the core in close proximity to the booster at the forward attachment location, the attachment flow provides sufficient perturbation to the cone-cylinder flow to make it unsteady. Thus, the combination of a marginally stable cone-cylinder flow perturbed by the forward attachment hardware flow produces an unsteady flow condition. In the attachment region shown, the shock structure is pulsing from side to side in the $\pm \mathrm{Z}$ direction. A better description of the pulsing nature of the flow can be seen in the surface streamtraces shown in Figure 14. While a lengthy time span is shown here, the change in pressure shown in Figure 11 is more representative.

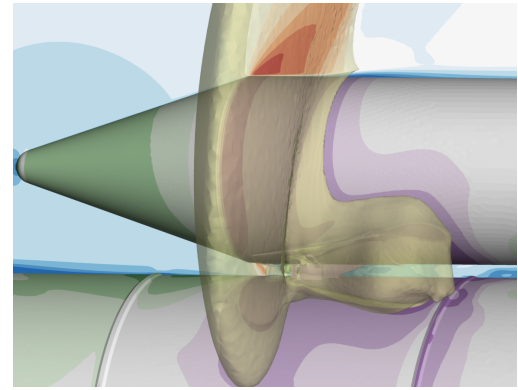

(a) Time step \#1.

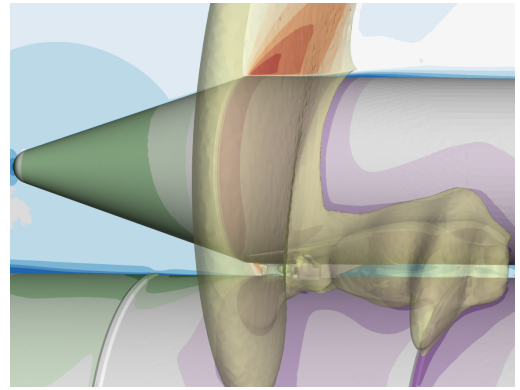

(b) Time step \#2.

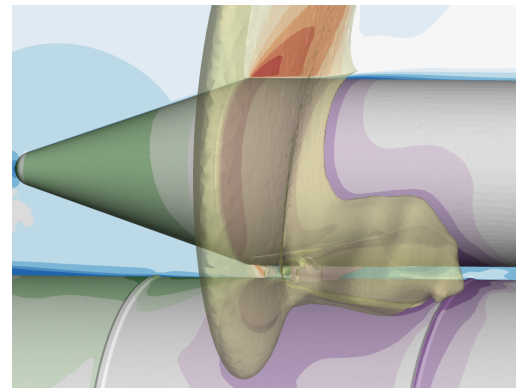

(c) Time step \#3.

Figure 13. Shock structures extracted at Mach=1 near forward attachment.

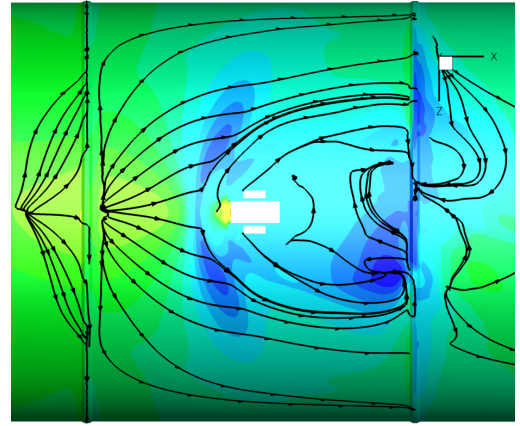

(a) Time step \#1.

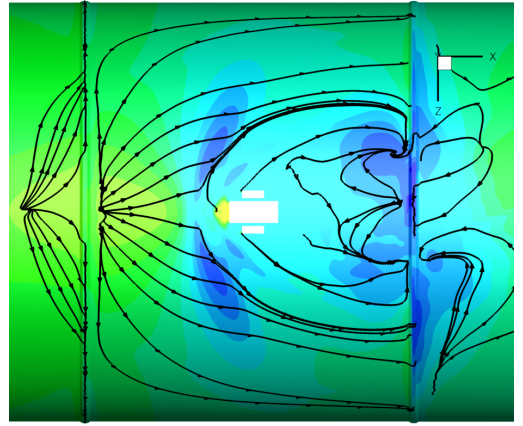

(b) Time step \#2.

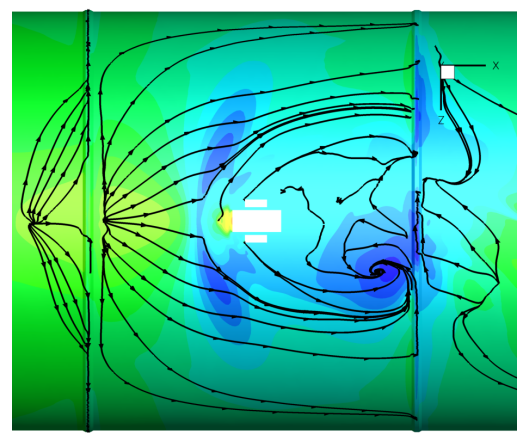

(c) Time step \#3.

Figure 14. Surface streamtraces with pressure contours around the forward attachment.

Figure 14 shows an oscillatory change in a low pressure condition in the wake of the forward attachment. The dark blue color indicating low pressure shifts from side to side, perturbing the cone-cylinder booster flow sufficiently to produce a consistent unsteady flow. Notable in this flow is that the low pressure location travels from side to side, producing a wave that propagates down the stack between the booster and the core. 


\section{VIII.A. Sensitivities}

To accurately and efficiently compute the variety of cases needed for this study, sensitivity of time step and mesh size had to be determined. Most computations were performed on the Pleiades supercomputer at NAS, which has in excess of 165,000 cores, enabling a multitude of cores to be employed for a time-accurate, transonic, booster-interaction computation. However, the FUN3D software has a general requirement of no less than 12,500 points per computing core without suffering from communications swamping the computations. Thus, there is a threshold of time required to obtain a solution. While it would be ideal to use the smallest time step and largest number of nodes that could fit onto Pleiades for highly accurate computations, such work could not be completed in a reasonable number of calendar days. Thus, grid and time step sensitivity analyses were performed in order to assess their effects on accuracy of the computation, in terms of capturing the peak power. This peak power was used as a reference point because it is clearly identifiable in the PSD.

\section{VIII.A.1. Time Step Sensitivity}

A time step sensitivity analysis was performed using the baseline mesh for the cases computed in this study. At the outset of the computing, rapid analysis methods were used, such as changing the time step in a solution after 20,000 time steps had been computed. No time steps were ignored after the changing of the time step, as would be done to get a better estimate of the best time step to use. Since a general time step to use was unknown, this quick method provided some guidance on the time step to use for maintaining accuracy and using as few resources as possible. As shown in Figure 15(a), increasing the non-dimensional time step produced a phase shift in the unsteady pressures as evidenced by the side force coefficient $\left(C_{y}\right)$ per simulation time elongating as simulation time increases. Decreasing the non-dimensional time step below the nominal value of 0.003 showed diminishing effects; and although a slight change in phase is noted between the non-dimensional time steps of 0.002 and 0.004 , computing at 0.003 is 50 percent faster than at 0.002 . Figure 15(b) shows a detailed investigation of the time sensitivity by using the unsteady surface pressure

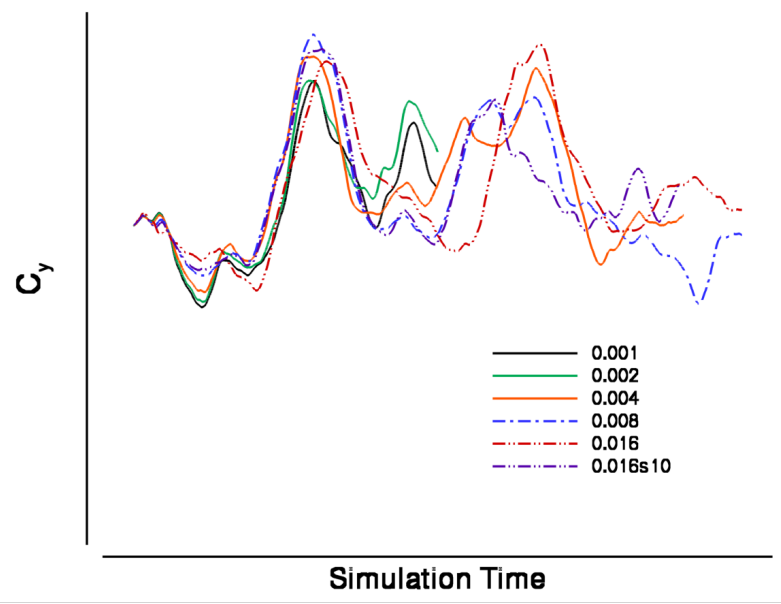

(a) Side force.

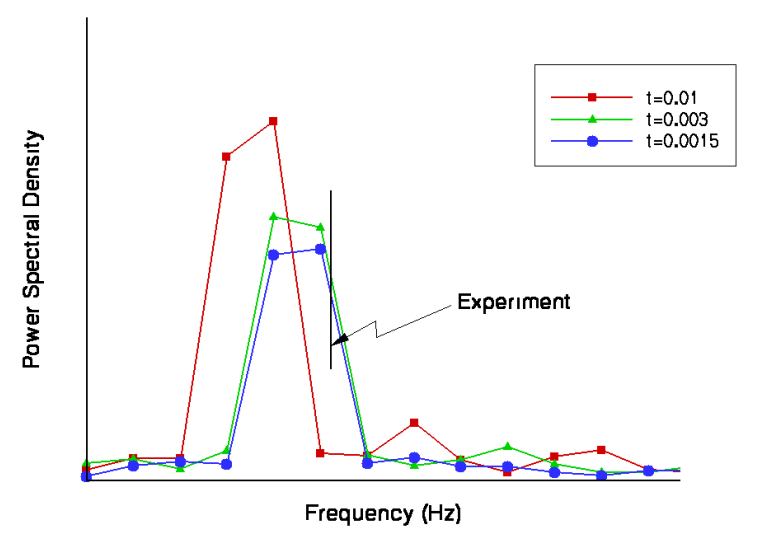

(b) Power spectral density.

Figure 15. Frequency phase shift due to changing the non-dimensional time step.

environment. In Figure 15(b), the symbols represent estimated frequency data. To obtain this plot, more than 160,000 time steps were computed for each case (each non-dimensional time step value). Only a few points on the vehicle identified the general frequency where the unsteady pressures had the most impact on the configuration. A PSD analysis of the frequencies for different time steps was performed. As can be seen in Figure 15(b), as the time step increases, frequency decreases, which is synonymous with the elongation of the side force frequency in Figure 15(a). As the non-dimensional time step is decreased, the differences diminish. While ever decreasing time steps are desirable, the smaller the time step, the longer a computation must be performed to accumulate sufficient data for analyses. Thus, a non-dimensional time step of 0.003 was selected for this study. 


\section{VIII.A.2. Mesh Size Sensitivity}

Another sensitivity evaluated was mesh density. To capture the boundary layer gradients accurately, approximately 50 prismatic cells extending orthogonally from the wall were used. In these meshes, a single cell size was used to provide a characteristic length for the remainder of the mesh. Figure 16 shows three different density meshes used in the characterization of the mesh sensitivity.

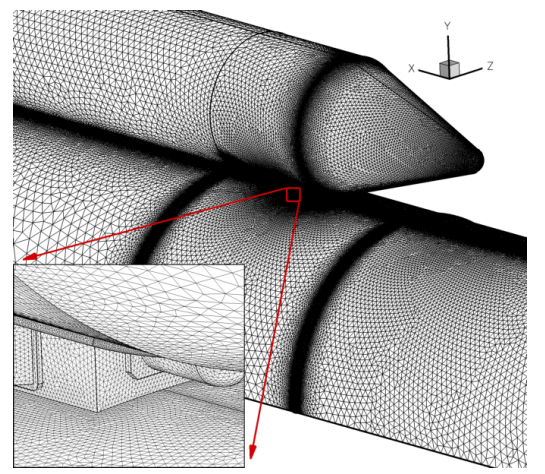

(a) Coarse (20M).

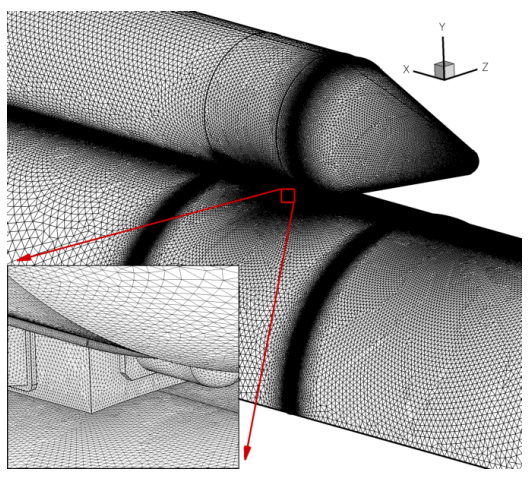

(b) Medium (baseline - 35M).

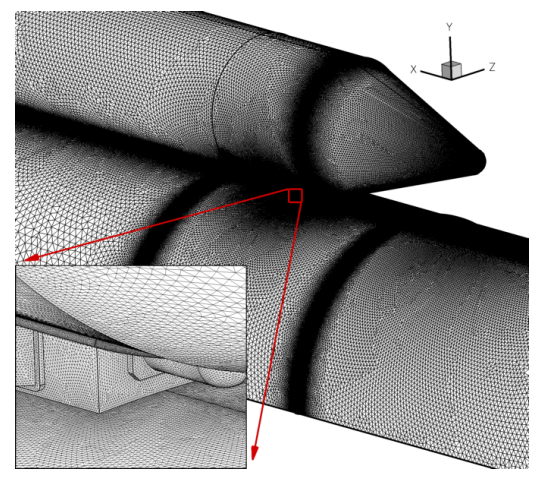

(c) Fine (59M).

Figure 16. Mesh density variation for a sensitivity analysis, with the medium mesh as the baseline density and node count in parenthesis.

The baseline characteristic scale for the reference cell size is defined to be 1 . This scale factor is based on resolving the smallest feature of the geometry being analyzed, and serves as a reference for the sensitivity study. Sources for the mesh generation were scaled based on this characteristic length. By referencing the characteristic cell size to define all other sources, mesh size is controlled by a single variable, the reference cell size. Increasing the scale produced fewer nodes in the mesh while reducing it produced increased node counts. Because the same sources were scaled by the characteristic scale, all generated meshes are within family; they are related to one another in cell distribution, mesh density, and overall mesh quality. In Figure 16, the coarse mesh has a scale factor of 1.3 , whereas the fine mesh had a scale factor of 0.8 . Using these meshes to compute the unsteady surface pressure fields, the mesh sensitivity is expressed in a PSD plot in Figure 17.

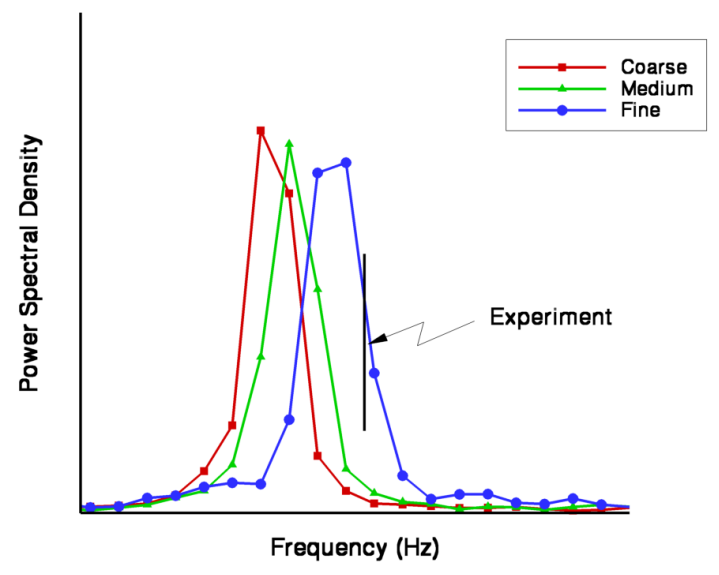

Figure 17. Solution accuracy based on a grid sensitivity analysis.

Figure 17 shows that as the grid is refined, the peak PSD is closer to the experiment. Further refinement showed improved accuracy but only marginally. This suggests that increasing the grid resolution improves agreement with experimental data, however, as the mesh becomes larger, it would also result in a mesh density that would be impractical for computations. The purpose of this study was to determine what is required to perform these computations. The performed work identified that large meshes with long run 
times are needed to get accurate data. Thus, the use of the baseline mesh size of 35 million nodes was deemed sufficient for the bulk of the computations because the results were sufficiently close to experimental data. The baseline mesh was the medium density mesh, shown in Figure 16. Changes in Reynolds number and Mach number at this grid resolution were evaluated. Increasing Reynolds number showed no appreciable differences in frequency shift towards experimental data and are not published. However, increasing Mach number showed better agreement with experimental data and were published. ${ }^{10}$

\section{VIII.B. Data Analyses}

Generally, the flowfield structure is very complex, particularly in the attachment hardware regions. The forward attachment region has a greater impact on the designs of the SLS because the current computation predicts an unsteady pressure environment, which leads to a buffet condition. To understand the extent of the buffet condition, several post-processing analyses were performed, including mesh density and time step sensitivities, comparisons of $C_{p, r m s}$ or differential pressure between wind tunnel data and CFD, analyses of the unsteady pressure frequencies including FFTs, histograms, and coherence. The sensitivities were discussed above, while the remaining analyses are explained in greater detail in the following sections.

\section{VIII.B.1. $C_{p, r m s}$}

Comparison of the root mean square surface pressure values between CFD and the experiment is made by collecting data at points in the CFD model, which are identical in location to those on the wind tunnel model. Due to model limitations, 237 pressure taps were measured on the wind tunnel model, while CFD monitor points totaled 619. The CFD monitor points occur at axial locations that correspond to those where pressure guages were installed on the model. The CFD points are spaced every $22.5^{\circ}$, where such density is not identical on the wind tunnel model. The wind tunnel model had fewer pressure taps at some X-stations (i.e., axial locations), having a separation of every $45.0^{\circ}$ or every $90.0^{\circ}$. Thus, the wind tunnel model does not have as dense a collection of pressure taps, as shown in Figure 18. Since half body computations were performed, the number of points compared between the CFD and wind tunnel data numbered 169. The remaining 41 points are on the side of the full body stack that was not computed.

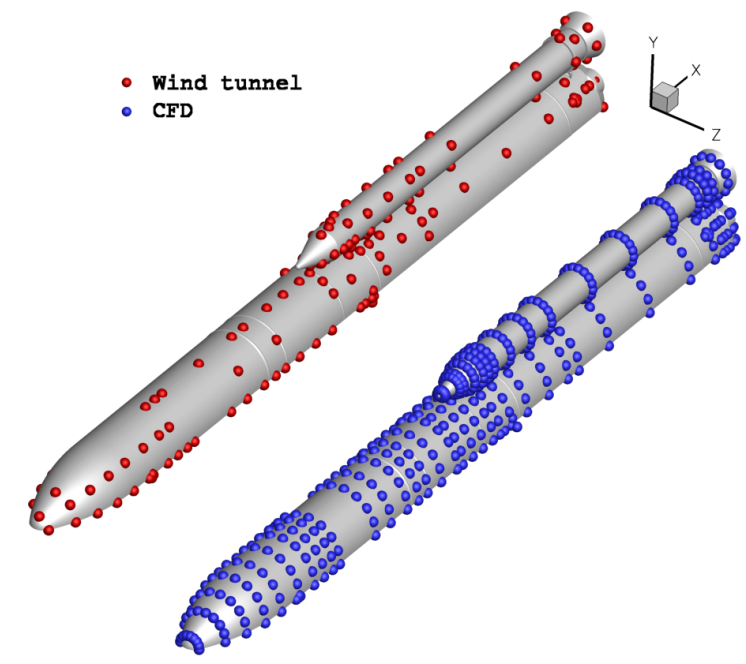

Figure 18. Pressure taps and monitor points used on the wind tunnel and CFD models, respectively.

Using inverse distance interpolation along the surface of the SLS, Figure 19 shows the $C_{p, r m s}$ flooded contours. In these plots, the overall geometry is shown in the upper left with a lower right magnified region showing the core without the booster and attachment geometry. The peak $C_{p, r m s}$ location was the same in both the wind tunnel and CFD monitor point data sets, downstream of the forward attach hardware on the centerline between core and booster. Interrogating the entire CFD surface, the peak $C_{p, r m s}$ location was shifted slightly aft by about 0.2 percent of model length, still on-centerline. This highlights one benefit of CFD simulation, namely higher spatial resolution than is possible with a wind tunnel model. Although the 
location for peak $C_{p, r m s}$ were the same between CFD and wind tunnel data sets, the peak values for $C_{p, r m s}$ in the CFD are lower. The lower $C_{p, r m s}$ values could be attributed to simplifications made to the CFD model in order to obtain a usable mesh for computing, noise in the tunnel, and model vibration not considered in the CFD simulations. Similar differences within the cross-section of the forward attach hardware where three rectangular holes are indicated in the magnified core plot can be seen. But the regions of greatest unsteady-pressures are qualitatively similar.

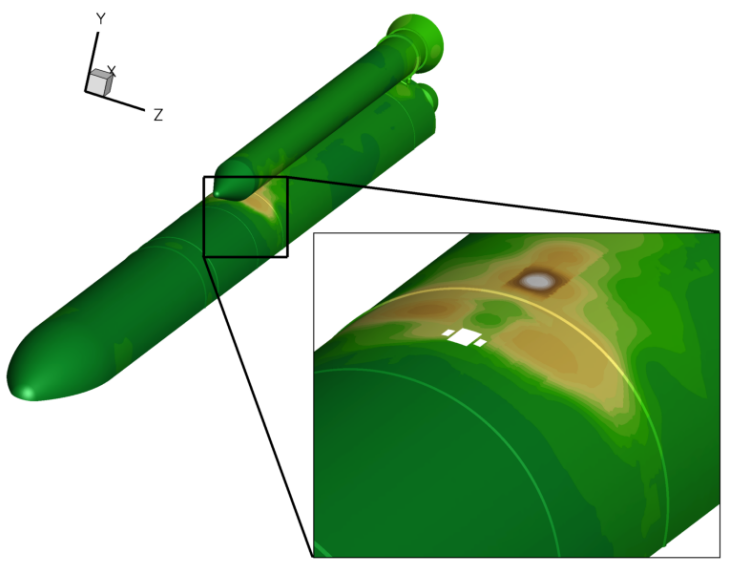

(a) Wind tunnel data.

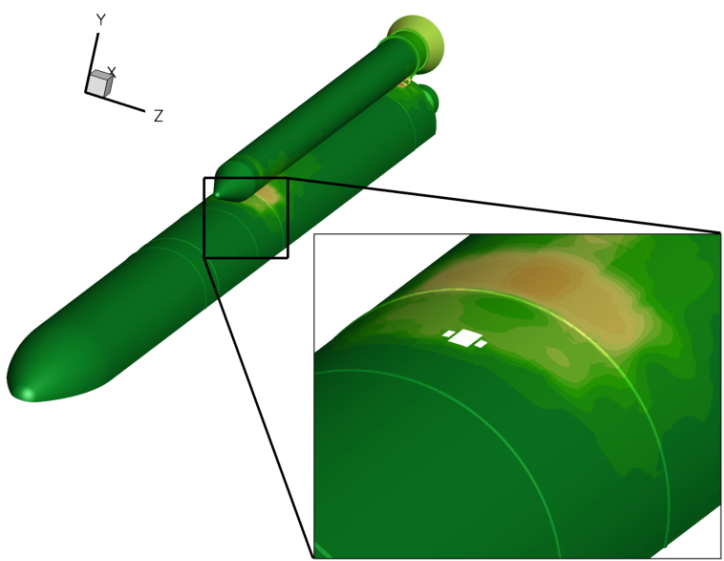

(b) CFD data.

Figure 19. $C_{p, r m s}$ plots of wind tunnel and CFD data.

\section{VIII.B.2. Histograms}

Another side-by-side comparison between the CFD flow solution and the wind tunnel data is comparing histograms of the pressure content. From FUN3D, the pressure data collected during the computations are reformulated to match the wind tunnel differential pressure $(\Delta P)$ by using Eq. (3). Note that only the locations in the vicinity of the highest $C_{p, r m s}$ region are considered. Figure 20 shows the particular points, noting the port numbers for reference to the following data. Five points encompass the largest change in pressure, including $89,99,100,101$, and 112, and were chosen to determine how well CFD is matching the wind tunnel measurements.

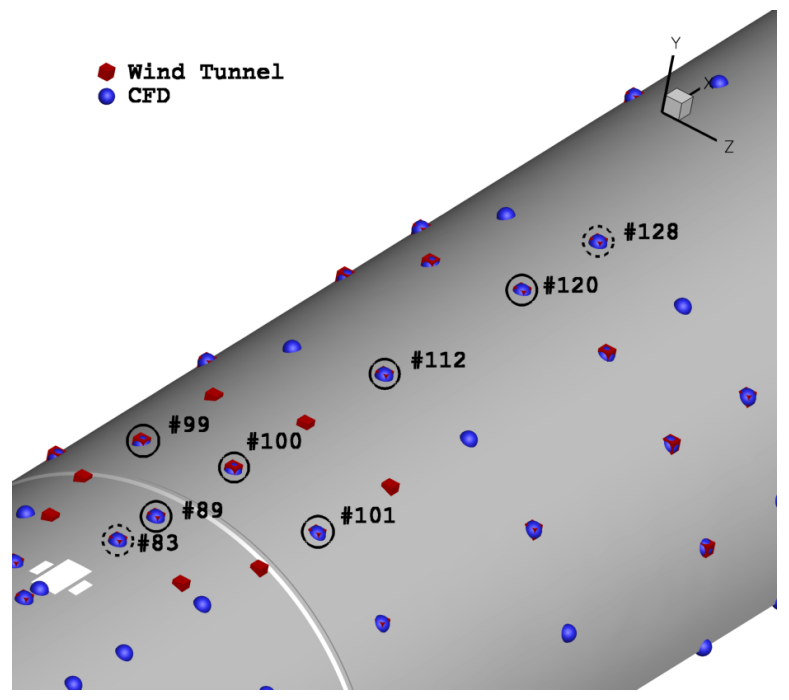

Figure 20. Pressure ports (taps solid circled) used for histogram and PSD analyses. 
Peak $C_{p, r m s}$ occurs at port 100 , which is central to the exploration of the data, both measured and computed. The CFD was computed at a sampling rate of close to $520 \mathrm{kHz}$. However, the wind tunnel data acquisition system collected data at $9.6 \mathrm{kHz}$. The pressure content of the CFD is approximately 54 times denser than the wind tunnel because of the smaller non-dimensional time step to accurately compute the flowfield. Thus, there are three plots of the histograms, normalized by the number of samples, for each data point shown in Figures 21 and 22 representing the five pressure ports ranging from 89 to 112, respectively. The plots include the wind tunnel data, the CFD sampled down to the same sampling rate as the wind tunnel, and the complete sample of the CFD to determine if the CFD is capturing additional phenomena that the sampling of the wind tunnel may not be able to resolve. All plots were generated with the same differential pressure range, consisting of 100 bins for the range, and the same percentage count axis range, except for port 89 . The count percentage range for port 89 is expanded by $50 \%$ to capture the entire plot.

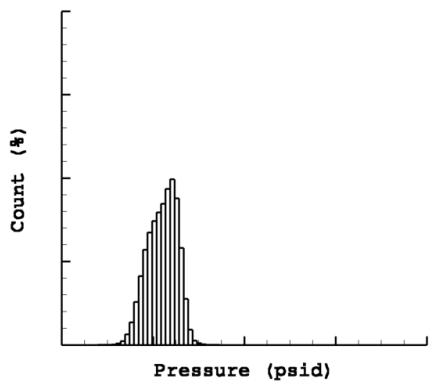

(a) Port 89 wind tunnel data.

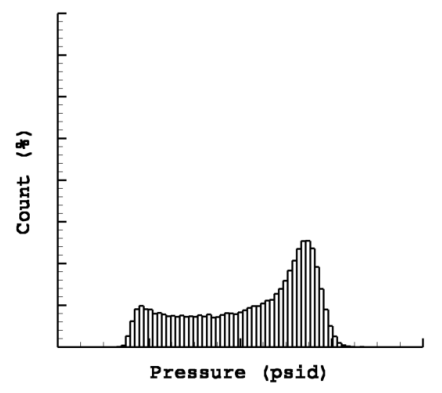

(d) Port 100 wind tunnel data.

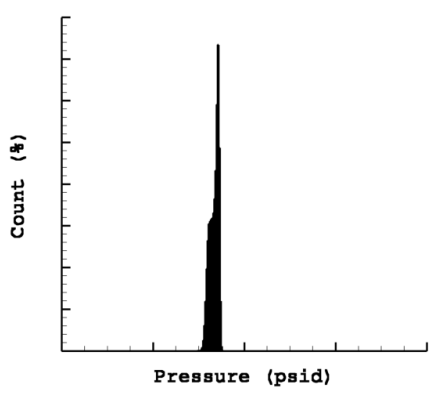

(g) Port 112 wind tunnel data.

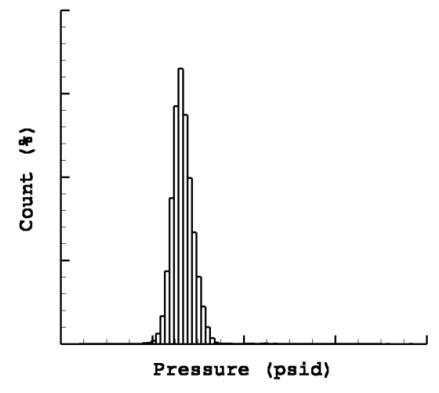

(b) Port 89 complete CFD data.

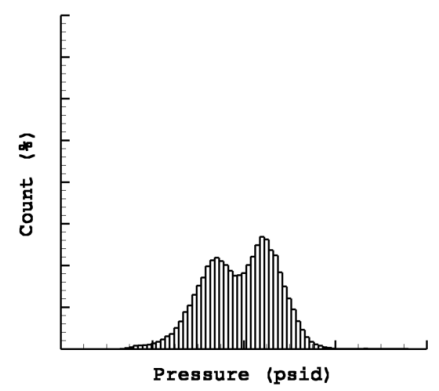

(e) Port 100 complete CFD data.

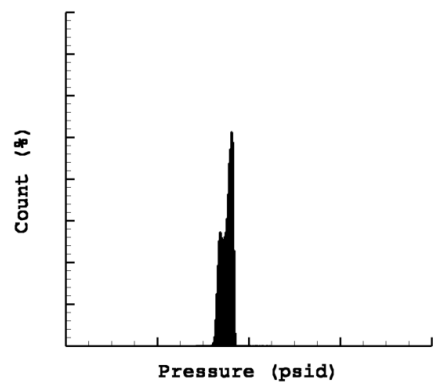

(h) Port 112 complete CFD data.

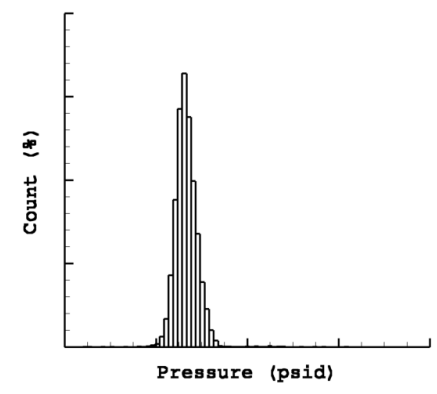

(c) Port 89 down sampled CFD data.

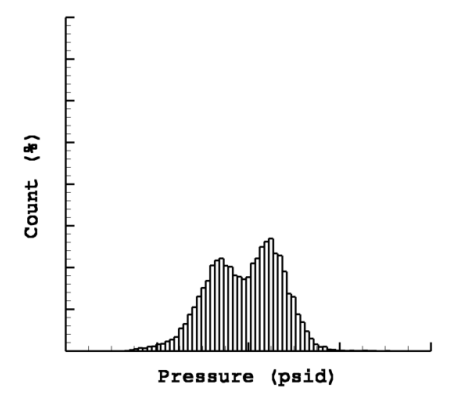

(f) Port 100 down sampled CFD data.

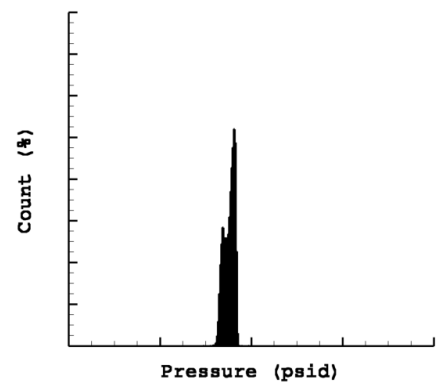

(i) Port 112 down sampled CFD data.

Figure 21. Histograms of surface pressure along center-line. 


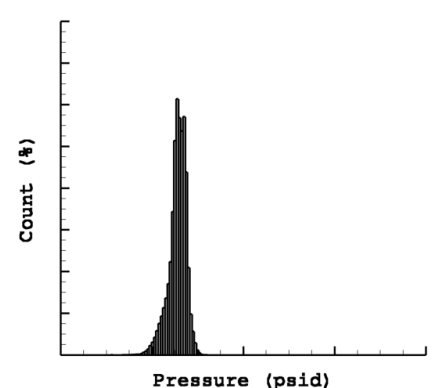

(a) Port 99 wind tunnel data.

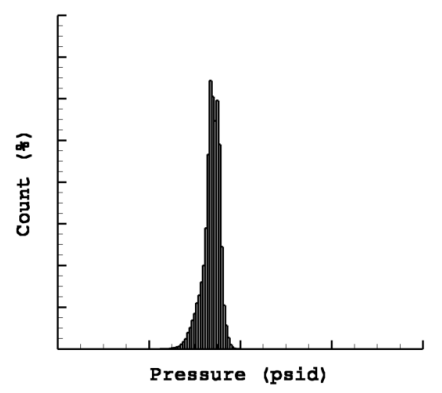

(d) Port 101 wind tunnel data.

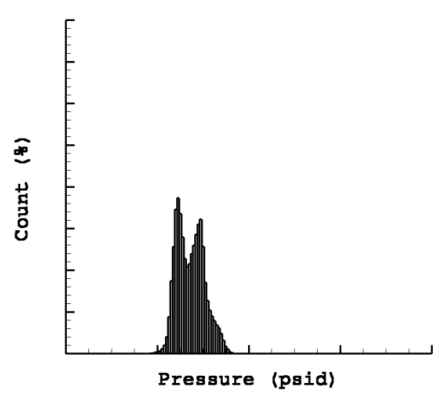

(b) Port 99 complete CFD data.

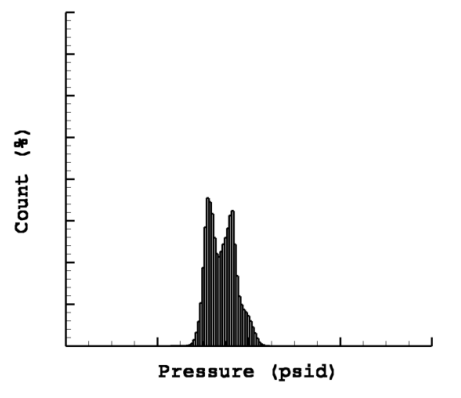

(e) Port 101 complete CFD data.

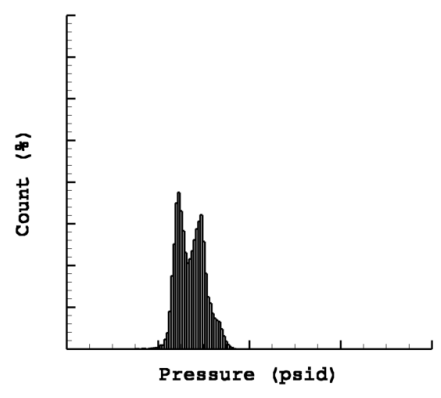

(c) Port 99 down sampled CFD data.

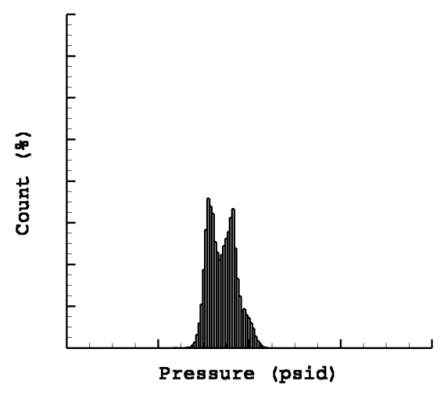

(f) Port 101 down sampled CFD data.

Figure 22. Histograms of surface pressure for off center-line.

Generally, consistent trends are present in most comparisons between wind tunnel and CFD data, but the histograms are not identical. For port 89, the histogram shapes are similar, but the CFD distribution is taller and more narrow. The wind tunnel data shows an expanded pressure range (indicative of large variations in pressure) whereas the CFD range is smaller for the bulk of the data. This trend holds true for most of the histograms except port 112 (indicative of a more constant pressure). At port 99 in Figure 22, there is a bi-modal signature in the CFD, and a slight indication of such in the wind tunnel data, though the range of the plot does not easily enable identification of the wind tunnel double peak. This feature, indicative of an alternating flow, ${ }^{36}$ holds true for port 101 as well. For the center-line data at port 100, where the peak unsteady-pressure occurs, there is a bi-modal signature, but the wind tunnel data shows an expanded "valley." This is troublesome, but may be the result of geometric differences as well as noise in the wind tunnel. Conversely, for the downstream center line port 112, the histogram shows a very consistent trend, both in shape and pressure range. Down sampling has very little effect in these evaluated ports, indicating there is no secondary physical phenomenon occurring that the wind tunnel measurements did not capture. While the peak $C_{p, r m s}$ port 100 does show an expanded range between the two pressure peaks, the remaining ports show consistent trends. Although these histograms do not match identically, the shapes are similar indicating the CFD has captured the physical phenomenon in the flowfield. 


\section{VIII.B.3. Power Spectral Density Estimates}

As noted in the Post-Processing section, Welch's method is used to evaluate the frequency content of the surface pressures collected in the wind tunnel and the CFD. All surface pressures for the CFD and the wind tunnel data were compared at the same frequency resolution. Using Hamming windowing, ${ }^{37,38}$ the power spectral density of pressure was generated for model scale frequency bins of $1.0 \mathrm{~Hz}$, and they are shown in Figure 23 for the six monitor points shown in Figure 20.

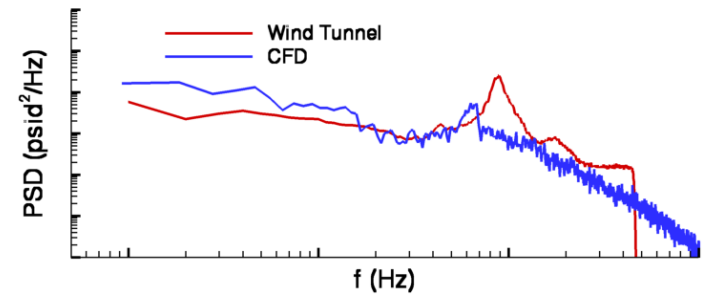

(a) Port 89.

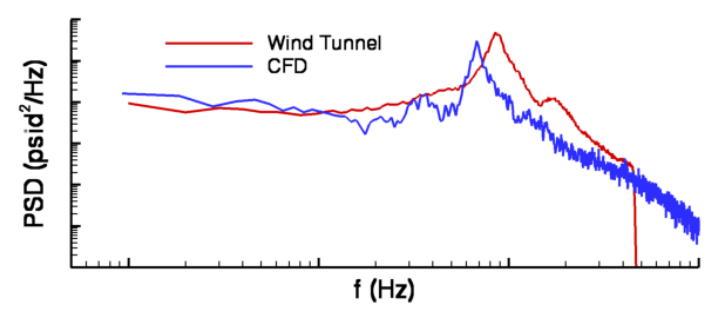

(c) Port 100.

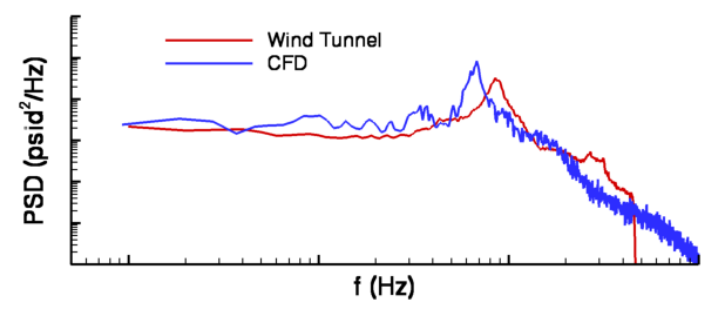

(e) Port 112.

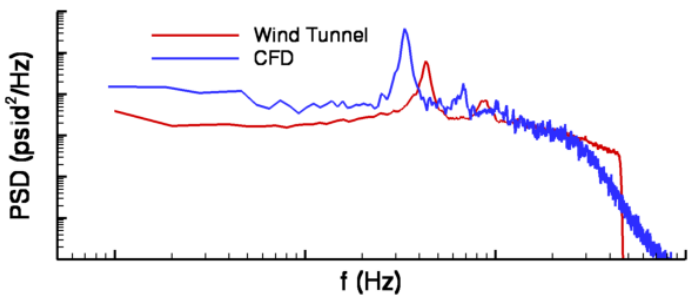

(b) Port 99.

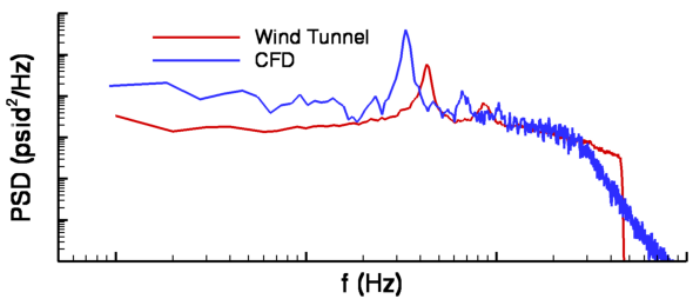

(d) Port 101.

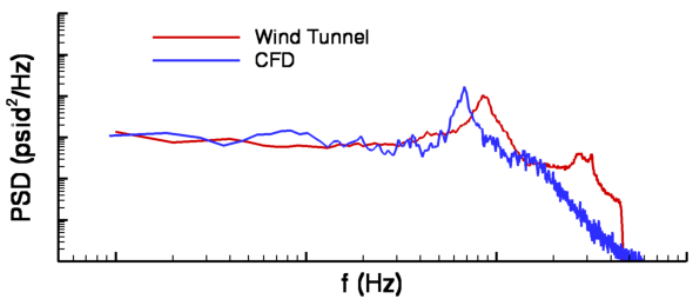

(f) Port 120.

Figure 23. Power spectral density of wind tunnel and CFD data for near peak $C_{p, r m s}$.

As can be seen in Figure 23, the CFD, shown as the red line on the plot, under-predicts the dominant frequency by approximately 20 percent at all the points in the vicinity of the peak unsteady-pressures. Differences may be because of the geometric simplifications made to generate the CFD unstructured meshes, in addition to mesh densities as identified in the sensitivities. Both the histograms and FFT analyses do suggest that the modified DDES model, which is implemented in the FUN3D CFD algorithm is capturing the physics, however, it too may be a source of differences. Differences aside, the comparison does show the DDES model is a useful algorithm to predict an unsteady pressure environment.

As discussed previously, one of the reasons for an averaged periodogram method such as Welch's method is to reduce the variance in the power estimates at the various frequencies. As the numbers of windows is increased (increased number of averages), the variance is reduced. Figure 24a shows the PSD estimate for wind tunnel data for different numbers of windows. Since the window size, and thus frequency resolution, was fixed, increasing the number of windows is synonymous with increasing data collection time. As highlighted by point 'B', approximately 200 windows are necessary to converge the power estimates. A similar analysis for the CFD is presented in Figure 24b. Again, a large number of windows is needed, which is synonymous with a large time record, for a reasonable frequency resolution. Additionally, these analyses are important because they provide a means to determine the simulation time needed to converge an unsteady CFD computation. These plots suggest that sufficient time has been utilized. 


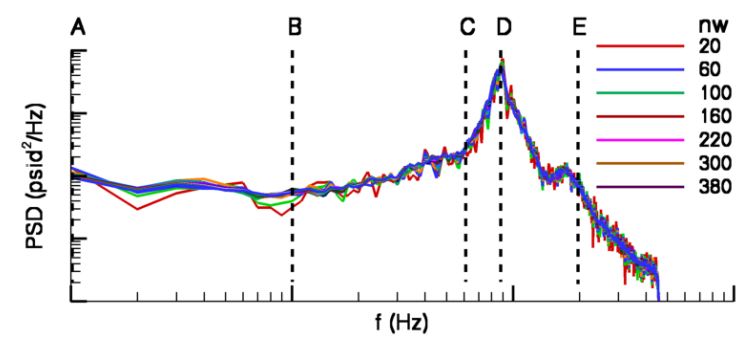

(a) Wind tunnel.

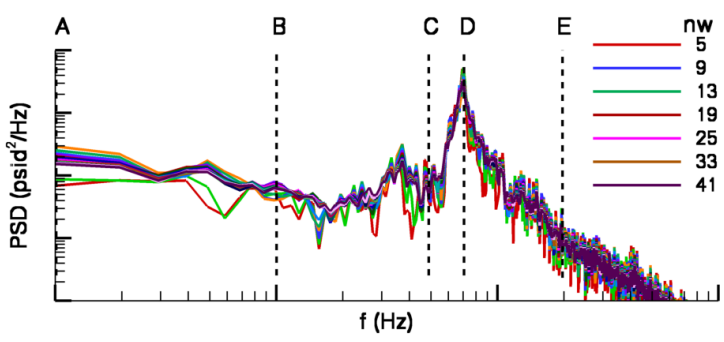

(b) CFD.

Figure 24. Power spectral density distribution for frequency based on the number of windows used.

One of the goals of this work was to determine how much time was required to be computed for the flowfield to get a consistent identification of the frequencies that can contribute to a buffet condition through the modeling of the unsteady-pressures. Probing the PSD data for specific frequencies at the locations shown in Figure 24 can provide this information. In Figure 25, the convergence of different frequencies is shown for both CFD and wind tunnel data. Based on the number of windows with a constant number of samples per window, it is clear that CFD nearly converges within 0.8 seconds or 40 windows of computed time, while the wind tunnel data requires at least 5 seconds of data, or 350 windows. The extended run time for the wind tunnel can be attributed to additional sources of errors not modeled in the CFD simulation. Some of these can include noise in the tunnel, flow differences over time, and model vibration. Thus, to obtain an accurate estimate of the PSD in a CFD simulation for this study, approximately one second of computed time was needed. Conversely, the wind tunnel data would support a higher frequency resolution, using a smaller frequency bin, with the 5 seconds of data. So the frequency resolution to use is important, but remains ill-defined.

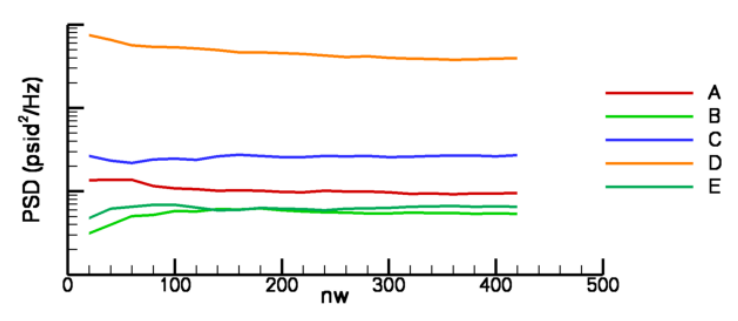

(a) Wind tunnel.

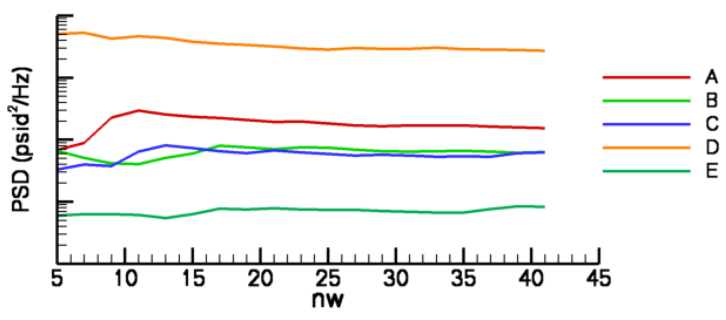

(b) CFD.

Figure 25. Power spectral density convergence for different frequencies in wind tunnel and CFD data.

\section{VIII.B.4. Coherence}

$C_{p, r m s}$, histograms, and power spectral densities of the surface pressures from an unsteady CFD solution, and wind tunnel test results provide an analysis of discrete locations on the SLS. Another measure, coherence, evaluates spatial effects along the vehicle surface. As discussed previously, coherence is a measure of signal similarity or correspondence between two points. For the SLS vehicle, referring to Figure 26, the signals between port 100 and ports 112, 120, 128, 136, 141, 146, 155, and 171 are evaluated for coherence.

The coherence between port 100 and a downstream location, port 112, is shown in Figure 27. The dominant frequency from Figure 23c is at point E. In Figure 27, the coherence at this frequency is approximately 0.95 , suggesting that these two points are clearly coherent.

As done with the power estimates, an evaluation of the required simulation time to converge the coherence estimates was done. As seen in Figure 27b, the coherence estimate at a given frequency may converge more slowly than the power estimates, particularly when the coherence is low. Frequencies at points A through $\mathrm{E}$ are evaluated, and shown in Figure 28. For the dominant frequency at point E in the CFD and D in the wind tunnel, the coherence is near 1.0 for all window counts (number of windows), both for CFD and wind 


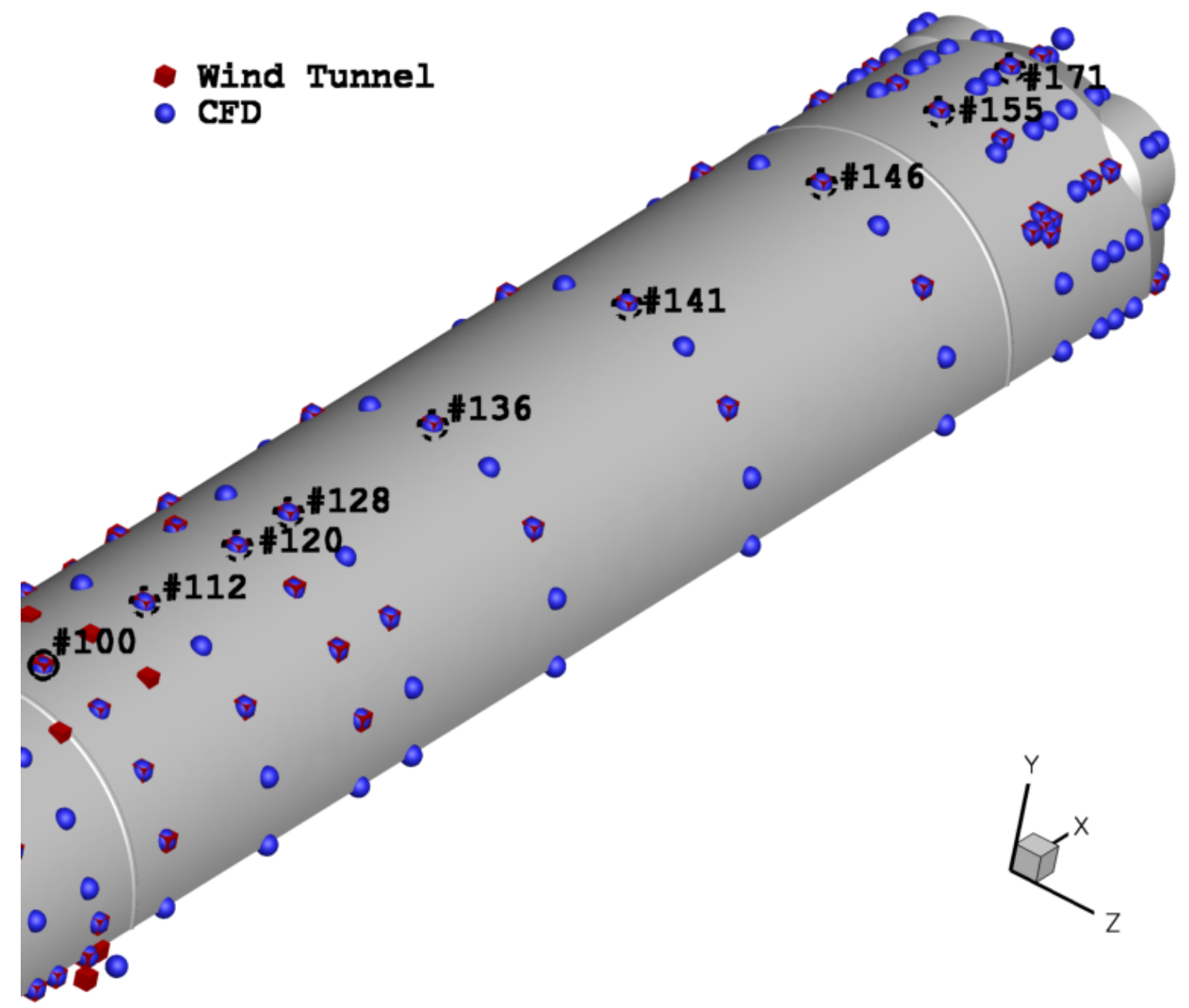

Figure 26. Pressure ports used for coherence analysis.

tunnel data. Thus, highly coherent frequencies are captured quickly in this analysis, while less coherent frequencies require significantly more simulated time to resolve. The frequency at point $\mathrm{C}$ suggests a nonconvergent state for CFD based on the number of windows. The value of 0.44 at the maximum number of windows suggests that at this frequency, coherence may not be obtainable. It is also possible that additional simulation time is needed to converge to a consistent coherence. Also note that approximately 200 windows are needed for the experimental data to get a convergence of coherence. Frequencies lower and higher show better convergence to a constant coherence. By comparison to the PSD analysis in Figure 25, more simulated time is required to get a guaranteed convergence on the coherence with respect to the number of windows, when there is a lack of coherence.

Using the results from the longest time, where the most windows are used, the other ports along the SLS core were evaluated for coherence to the peak frequency location in the CFD data at point E. Based on the windowing analysis, the dominant frequency which occurs at port 100, is close to the same value at each pressure monitor point, as shown in Figure 29. The dominant frequency of the unsteady differential pressure is coherent from the forward attachment hardware to the end of the vehicle and explains the coherence peak for all the plots. Thus, from Figure 28, a driving frequency for a buffet condition along the core at this model scale occurs at point $\mathrm{E}$ in the CFD and D in the wind tunnel, and the oscillatory force produced by this frequency propagates along the core. The relatively good agreement in the coherence plots between CFD and experiment (away from the very lowest frequencies, is an indication that the time relationship is correct between longitudinal stations on the vehicle. This is important as it implies the buffet loads, which are integrated loads applied at the various vehicle stations, will also be in the proper time relationship. 


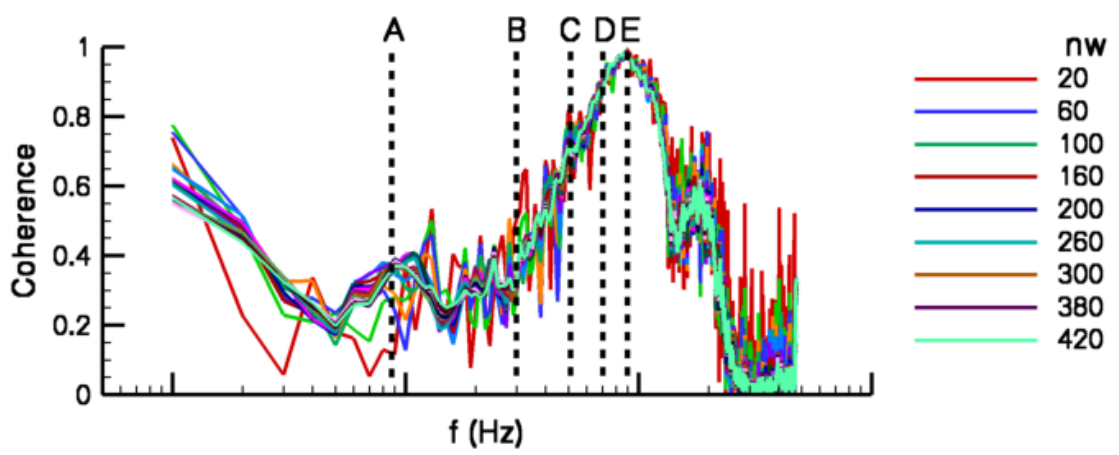

(a) Wind tunnel.

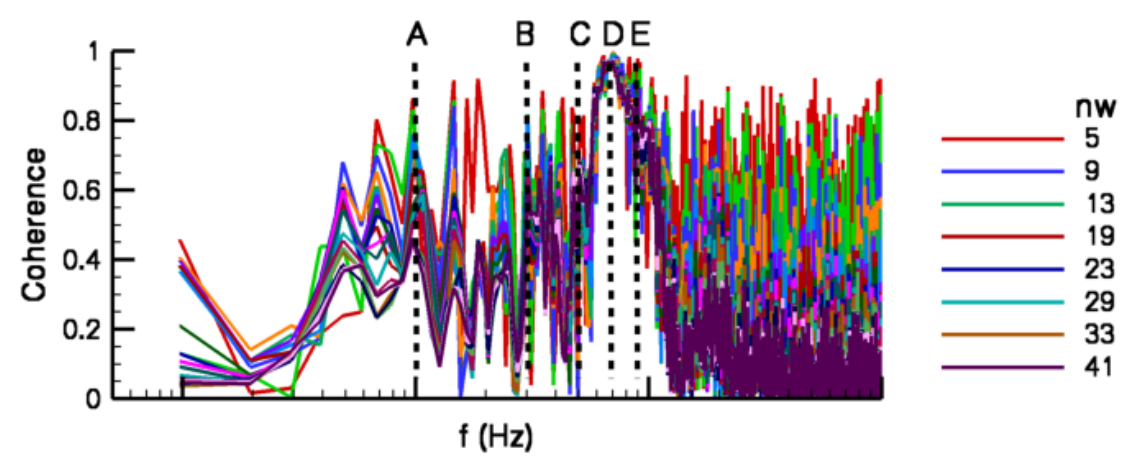

(b) CFD.

Figure 27. Coherence between ports 100 and 112 along SLS core for wind tunnel and CFD data.

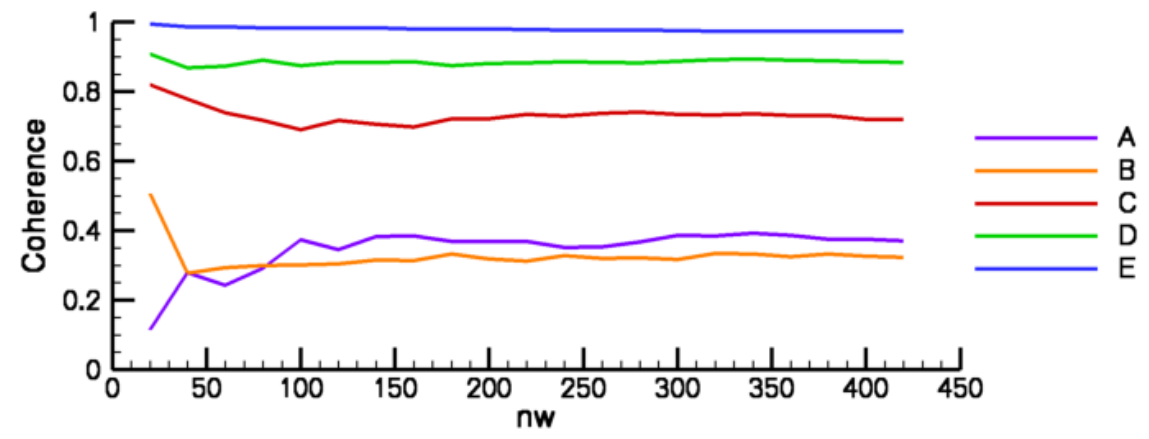

(a) Wind tunnel.

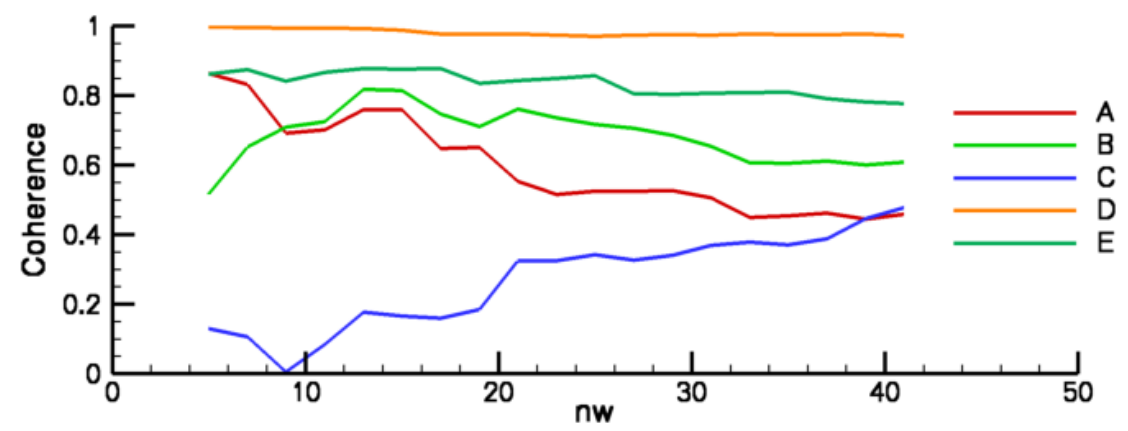

(b) CFD.

Figure 28. Coherence between ports 100 and 112 for different numbers of windows, comparing wind tunnel and CFD data. 


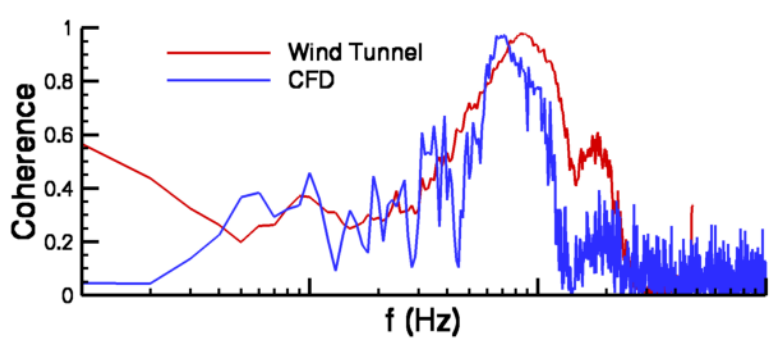

(a) Port 100 to 112 .

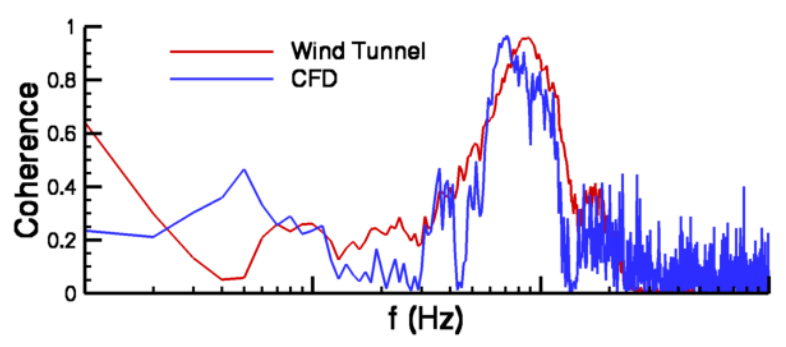

(c) Port 100 to 128 .

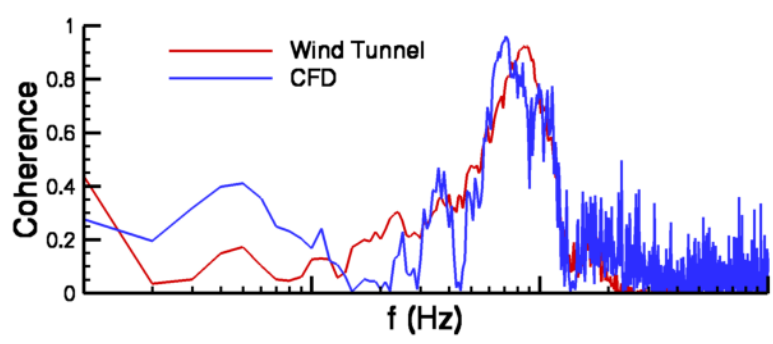

(e) Port 100 to 141 .

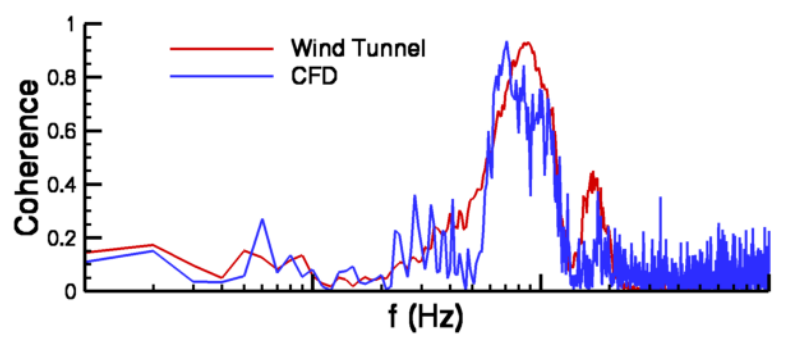

(g) Port 100 to 155 .

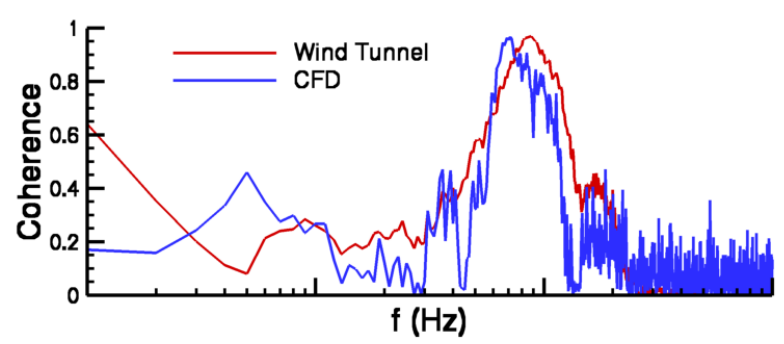

(b) Port 100 to 120 .

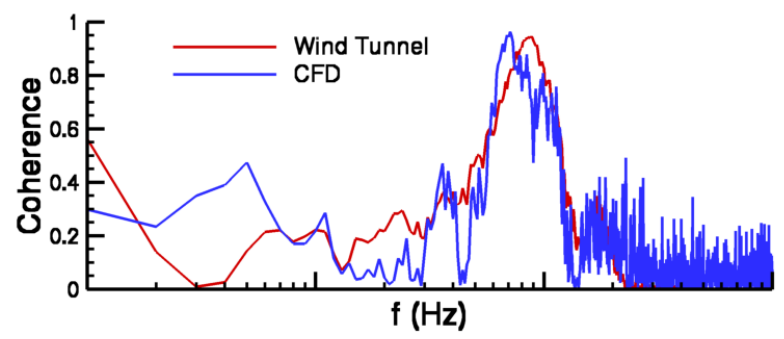

(d) Port 100 to 136.

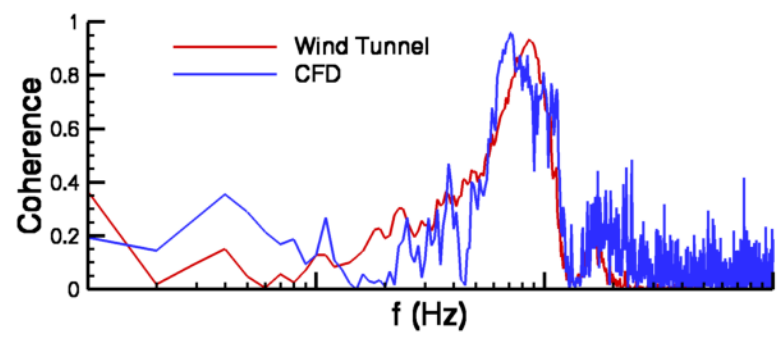

(f) Port 100 to 146.

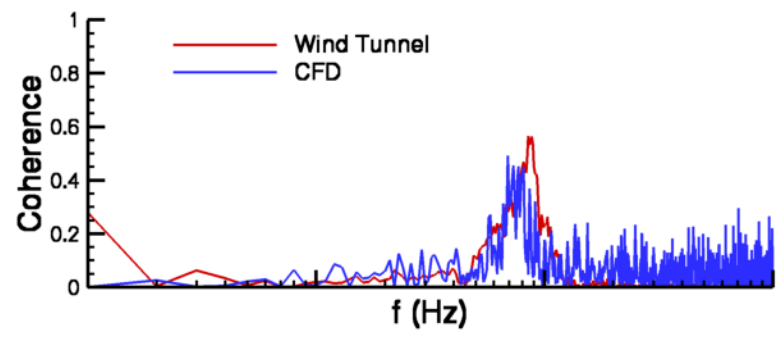

(h) Port 100 to 171.

Figure 29. Coherence between ports along SLS core from the peak pressure differential at port 100 . 


\section{Summary}

The Fully Unstructured Three-Dimensional flow solver (FUN3D), with a modified Delayed Detached Eddy Simulation method, was used to compute the unsteady flowfield over a Space Launch System configuration at transonic speeds. The solution identified an oscillatory pressure loading along the central core of the vehicle, which correlates well with wind tunnel data collected from a test of a 2.5 percent scaled SLS model in the unitary wind tunnel at NASA Ames Research Center. Comparisons of $C_{p, r m s}$ between both experimental and computed data sets indicate that the peak $C_{p, r m s}$ location was the same, and the frequency of peak power spectral density was within 25 percent between both data sets. Comparisons of histograms of unsteady pressures indicate that FUN3D captures the physical phenomenon of the flowfield, while frequency domain analyses indicate that the flow is in good agreement with wind tunnel data. Yet the analysis also indicates that using the current CFD data may not be sufficiently accurate to develop buffet forcing functions. While not the first time CFD has been used to model these flowfield conditions, this work may provide the basis for using computed data for detailed analyses of ascent buffet conditions for space flight vehicles.

\section{Acknowledgments}

This work was done in support of the NASA Engineering Safety Center (NESC), for the Space Launch System Program. The authors wish to acknowledge the guidance provided by John Blevins of NASA Marshal Space Flight Center, and Russ Rausch of NASA Langley Research Center. CAD geometries, provided by NASA Marshal Space Flight Center, were modified to develop solid manifold bodies for use with the mesh generation software, by Scott Brynildsen of ViGYAN and Ben Penatzer of ATI.

\section{References}

${ }^{1}$ Pritchett, V. E., Mayle, M. N., Blevins, J. A., Crosby, W. A., and Purinton, D. C., "Aerodynamic Tests of the Space Launch System for Database Development," AIAA Paper 2014-1256, January 2014.

${ }^{2}$ Niedermeyer, M. and Heidelberg, M., "Space Launch System NASA Research Announcement Advanced Development Office," http://www.nasa.gov/pdf/623766main_8143_Singer_AD_industry_day-021312_FINAL3.pdf, February 2012, NASA Head Quarters Space Launch System (SLS) Program Overview [cited 11 December 2014].

${ }^{3}$ Piatak, D. J., Sekula, M. K., and Rausch, R. D., "Comparison of Ares I-X Windtunnel Derived Buffet Environment with Flight Data," AIAA Paper 2011-3013, June 2011.

${ }^{4}$ Balakrishna, S. and Acheson, M. J., "Analysis of NASA Common Research Model Dynamic Data," AIAA Paper 20111127, January 2011.

${ }^{5}$ Engblom, W. A., "Numerical Simulation of Titan IVB Buffet Environment," Journal of Spacecraft and Rockets, Vol. 40, No. 5, 2003, pp. 648-656.

${ }^{6}$ Spalart, P. R., Deck, S., Shur, M. L., Squires, K. D., Strelets, M. K., and Travin, A., "A New Version of Detached-eddy Simulation, Resistant to Ambiguous Grid Densities," Theoretical and Computational Fluid Dynamics, Vol. 20, No. 3, 2006, pp. 181-195.

${ }^{7}$ Tsutsumi, S., Takaki, R., Takama, Y., Imagawa, K., Nakakita, K., and Kato, H., "Hybrid LES/RANS Simulations of Transonic Flowfield Around a Rocket Fairing," AIAA Paper 2012-2900, June 2012.

${ }^{8}$ Pain, R., Weiss, P. E., and Deck, S., "Zonal Detached Eddy Simulation of the Flow Around a Simplified Launcher Afterbody," AIAA Journal, Vol. 52, No. 9, February 2014, pp. 1967-1979.

${ }^{9}$ Spalart, P. R., "Young Person's Guide to Detached Eddy Simulation Grids," NASA CR 2001-211032, National Aeronautics and Space Administration, July 2001.

${ }^{10}$ Brauckmann, G. J., Streett, C. L., Kleb, W. L., Alter, S. J., Murphy, K. J., and Glass, C. E., "Computational and Experimental Unsteady Pressures for Alternate SLS Booster Nose Shapes," AIAA Paper 2015-yet to be assigned, January 2015.

${ }^{11}$ Cole, S. R., Noll, T. E., and III, B. P., "Transonic Dynamics Tunnel Aeroelastic Testing in Support of Aircraft Development," Journal of Aircraft, Vol. 40, No. 5, 2003, pp. 820-831.

12 "NASA Ames Unitary Plan Wind Tunnel (UPWT) Complex," http://www.nasa.gov/centers/ames/orgs/aeronautics/ windtunnels/11x11-wind-tunnel.html, [cited 28 October 2014].

${ }^{13}$ Kmak, F., "Modernization and Activation of the NASA Ames 11-By 11-Foot Transonic Wind Tunnel," AIAA Paper 2000-2680, June 2000.

${ }^{14}$ Kleb, W., Schauerhamer, D. G., Trumble, K., Sozer, E., Barnhardt, M., renee Carlson, J., and Edquist, K., "Toward Supersonic Retropropulsion CFD Validation," AIAA Paper 2011-3490, June 2011.

${ }^{15}$ Anderson, W. K., Gropp, W. D., Kaushik, D. K., Keyes, D. E., and Smith, B. F., "Achieving High Sustained Performance in an Unstructured CFD Application," NASA CR 2000-210080, January 2000.

${ }^{16}$ Biedron, R. T., Derlaga, J. M., Park, M. A., Hammond, D. P., Gnoffo, P. A., Carlson, J. R., Thomas, J. L., Lee-Rausch, E. M., White, J. A., Carpenter, M. H., Jones, W. T., Runsey, C. L., Vatsa, V. N., Derlaga, J. M., Wood, W. A., Kleb, W. L., O'Brien, Jr., D. M., Smith, M. J., and Lynch, C. E., "FUN3D Manual: 12.4," NASA TM 2014-218179, March 2014. 
${ }^{17}$ Vatsa, V. N. and Lockard, D. P., "Assessment of Hybrid RANS/LES Turbulence Models for Aeroacoustics Applications," AIAA Paper 2010-4001, 2010.

${ }^{18}$ Edwards, J. R., "A Low-Diffusion Flux-Splitting Scheme for Navier-Stokes Calculation," Computers and Fluids, Vol. 26, No. 6, July 1997, pp. 653-659.

${ }^{19}$ van Albada, G. D., van Leer, B., and Roberts, W. W., "A comparative Study of Computational Methods in Cosmic Gas Dynamics," Astronomy and Astrophysics, Vol. 108, No. 1, April 1982, pp. 76-84.

${ }^{20}$ Vatsa, V. N. and White, J. A., "Calibration of a Unified Flux Limiter for Ares-Class Launch Vehicles from Subsonic to Supersonic Speeds," Jannaf paper, April 2009.

${ }^{21}$ Spalart, P. R. and Allmaras, S. R., "A One-Equation Turbulence Model for Aerodynamic Flows," AIAA Paper 92-0439, January 1992.

22 "NX (Unigraphics) from Siemens PLM Software, Plano, TX," http://www.plm.automation.siemens.com/en_us/products/ $\mathrm{nx} /$, [cited 5 May 2014].

${ }^{23}$ Jones, W. T., "GridEx - An Integrated Grid Generation Package for CFD," AIAA Paper 2003-4129, 2003.

${ }^{24}$ Jones, W. T., "An Open Framework for Unstructured Grid Generation," AIAA Paper 2002-3192, 2002.

${ }^{25}$ Marcum, D. L. and Weatherill, N. P., "Unstructured grid generation using iterative point insertion and local reconnection," AIAA Journal, Vol. 33, No. 9, 1995, pp. 1619-1625.

${ }^{26}$ Marcum, D. L., "Efficient Generation of High-Quality Unstructured Surface and Volume Grids," Engineering With Computers, Vol. 17, No. 3, 2001, pp. 211-233.

${ }^{27}$ Peiro, J., Peraire, J., and Morgan, K., "FELISA Reference Manual and User's Guide, Volume I," University of Wales/Swansea Report CR/821/94, 1994.

${ }^{28}$ Perairee, J., Peiro, J., and Morgan, K., "Multigrid Solution of the 3D Compressible Euler Equations on Unstructured Tetrahedral Grids," International Journal of Numerical Methods Engineering, Vol. 36, No. 6, 1993, pp. 1029-1044.

${ }^{29}$ Parikh, P., Pirzadeh, S., and Löhner, R., "A Package for 3-D Unstructured Grid Generation, Finite-Element Flow Solutions, and Flow-Field Visualization," NASA CR 182090, September 1990.

${ }^{30}$ Löhner, R. and Parikh, P., "Three-Dimensional Grid Generation by the Advancing Front Method," International Journal of Numerical Methods and Fluids, Vol. 8, 1988, pp. 1135-1149.

${ }^{31}$ Haimes, R. and Follen, G., "Computational Analysis Programming Interface," Proceedings of the 6th International Conference on Numerical Grid Generation in Computational Field Simulation, edited by Cross, Eiseman, Hauser, Soni, and Thompson, July 1998, pp. 10-20.

32 "NASA's, High-End Computing Capability," http://www.nas.nasa.gov/hecc/\#url, [cited 28 October 2014].

${ }^{33}$ Biswas, R., Dunbar, J. A., Hardman, J. P., Bailey, F. R., Wheeler, L. F., and Rogers, S. E., "The Impact of High-End Computing on NASA Missions," IT Professional, Vol. 14, no. 2, IEEE Computer Society, March/April 2012, pp. 20-28.

${ }^{34}$ Welch, P. D., "The Use of Fast Fourier Transform for the Estimation of Power Spectra: A Method Based on Time Averaging Over Short, Modified Periodograms," IEEE Transactions on Audio Electroacoustics, Vol. AU-15, IEEE, June 1967, pp. $70-73$.

${ }^{35}$ Chevalier, H. L. and Robertson, J. E., "Pressure Fluctuations Resulting from an Alternating Flow Separation and Attachment at Transonic Speeds," Technical Documentary Report AEDC-TDR-63-204, Arnold Engineering Development Center, November 1963.

${ }^{36}$ Sekula, M. K., Piatak, D. J., and Rausch, R. D., "Analysis of Ares Crew Launch Vehicle Transonic Alternating Flow Phenomenon," AIAA Journal of Spacecraft and Rockets, Vol. 49, No. 5, September-October 2012, pp. $788-797$.

${ }^{37}$ Blackman, R. B. and Tukey, J. W., The Measurement of Power Spectra: From the Point of View of Communications Engineering, Dover Publications, Inc., 1958.

${ }^{38}$ Kanasewich, E. R., Time Sequence Analysis in Geophysics, The University of Alberta Press, 1973. 